\title{
NEW ANALYTICAL AND GEOMETRICAL ASPECTS OF THE ALGEBRAIC MULTIPLICITY
}

\author{
JULIÁN LÓPEZ-GÓMEZ, JUAN CARLOS SAMPEDRO
}

\begin{abstract}
This paper reveals some new analytical and geometrical properties of the generalized algebraic multiplicity, $\chi$, introduced in [7, 5] and further developed in [20, 23, 24. In particular, it establishes a completely new connection between $\chi$ and the concept of local intersection index of algebraic varieties, a central device in Algebraic Geometry. This link between Nonlinear Spectral Theory and Algebraic Geometry provides to $\chi$ with a deep geometrical meaning. Moreover, $\chi$ is characterized through the new notion of local determinant of the Schur operator associated to the linear path, $\mathfrak{L}(\lambda)$.
\end{abstract}

\section{INTRODUCTION}

This paper establishes some new properties of the generalized algebraic multiplicity introduced in [7, 5] and further developed in [20, 23, 24], denoted by $\chi$ in this paper. In particular, it reveals a hidden connection between $\chi$ and the concept of local intersection index of algebraic varieties, a central device in Algebraic Geometry. $\chi$ is a concept of multiplicity for general curves of linear Fredholm operators, $\mathfrak{L}(\lambda)$, which has shown to be pivotal in Bifurcation Theory and Nonlinear Analysis.

Classical Spectral Theory deals with special curves $\mathfrak{L} \in \mathcal{C}\left(\Omega, \mathcal{L}_{c}(U, V)\right)$ of the form

$$
\mathfrak{L}(\lambda)=\lambda I_{U}-K, \quad \lambda \in \Omega,
$$

where $U$ and $V$ are two $\mathbb{K}$-Banach spaces such that $U \subset V, \mathbb{K} \in\{\mathbb{R}, \mathbb{C}\}, \Omega$ is an open domain of $\mathbb{K}, \mathcal{L}_{c}(U, V)$ stands for the space of linear continuous operators that are compact perturbations of the identity map, $I_{U}$, and $K$ is a given compact operator. Adopting a geometrical perspective, Classical Spectral Theory studies the intersections of the straight line $\mathfrak{L}(\lambda)$ with the space of singular operators

$$
\mathcal{S}(U, V):=\mathcal{L}(U, V) \backslash G L(U, V),
$$

where $G L(U, V)$ denotes the space of invertible operators. In this context, $\lambda_{0} \in \Omega$ is said to be an eigenvalue of the straight line $\mathfrak{L}(\lambda)=\lambda I_{U}-K$ if $\mathfrak{L}\left(\lambda_{0}\right) \in \mathcal{S}(U, V)$, i.e., if $\lambda_{0} \in \sigma(K)$. Note that, in particular, these linear paths lie in the set of Fredholm operators of index zero, denoted in this paper by $\Phi_{0}(U, V)$.

More generally, Nonlinear Spectral Theory deals with general continuous paths in $\Phi_{0}(U, V), \mathfrak{L} \in \mathcal{C}\left(\Omega, \Phi_{0}(U, V)\right)$, generalizing the classical theory not only because it deals with arbitrary continuous curves, not necessarily straight lines, but also because these paths can lie in $\Phi_{0}(U, V)$ remaining outside $\mathcal{L}_{c}(U, V) \varsubsetneqq \Phi_{0}(U, V)$. In this general context, given a Fredholm path, $\mathfrak{L} \in \mathcal{C}\left(\Omega, \Phi_{0}(U, V)\right)$, a value $\lambda \in \Omega$ is said to be a generalized

2010 Mathematics Subject Classification. 47J10, 47J15, 58C40, $14 \mathrm{C} 17$.

Key words and phrases. Algebraic multiplicity, Nonlinear Spectral Theory, Schur complement, local determinant, bifurcation equation, local intersection index, Algebraic Geometry.

The first author has been supported by the Research Grant PGC2018-097104-B-100 of the Spanish Ministry of Science, Technology and Universities and by the Institute of Interdisciplinar Mathematics of Complutense University. The second author has been supported by PhD Grant PRE2019_1_0220 of the Basque Country Government. 
eigenvalue of $\mathfrak{L}$ if $\mathfrak{L}(\lambda) \notin G L(U, V)$. Then, the generalized spectrum of $\mathfrak{L}, \Sigma(\mathfrak{L})$, consists of the set of generalized eigenvalues, i.e.,

$$
\Sigma(\mathfrak{L}):=\{\lambda \in \Omega: \mathfrak{L}(\lambda) \notin G L(U, V)\} .
$$

Thus, as in the classical case, $\Sigma(\mathfrak{L})$ also consists of the intersection points of the curve $\mathfrak{L}$ with the singular manifold $\mathcal{S}(U, V) \cap \Phi_{0}(U, V)$. Naturally, for every compact operator $K$,

$$
\Sigma\left(\lambda I_{U}-K\right)=\sigma(K) .
$$

An important ingredient in Nonlinear Spectral Theory is the concept of generalized algebraic multiplicity, which assigns to every pair $\left(\mathfrak{L}, \lambda_{0}\right)$ with $\mathfrak{L} \in \mathcal{C}^{\infty}\left(\Omega, \Phi_{0}(U, V)\right)$ and $\lambda_{0} \in \Sigma(\mathfrak{L})$, an integer $\chi\left[\mathfrak{L}, \lambda_{0}\right] \in[1,+\infty]$ such that, whenever $\operatorname{dim} U=\operatorname{dim} V<\infty$,

$$
\chi\left[\mathfrak{L}, \lambda_{0}\right]=\operatorname{ord}_{\lambda_{0}} \operatorname{det} \mathfrak{L}(\lambda) .
$$

Although a number of constructions of $\chi\left[\mathfrak{L}, \lambda_{0}\right]$ had been done by Göhberg and Sigal [11], Magnus [25], Ize [15], Esquinas and López-Gómez [7], Esquinas [6] and Rabier [28], it was not until 2001, that Chapters 4 and 5 of López-Gómez [20] characterized whether all these generalized algebraic multiplicities were finite through the pivotal concept of algebraic eigenvalue. A generalized eigenvalue, $\lambda_{0} \in \Omega$, of a continuous path of Fredholm operators, $\mathfrak{L}(\lambda) \in \mathcal{C}\left(\Omega, \Phi_{0}(U, V)\right)$, is said to be $\kappa$-algebraic if there exist $\varepsilon>0$ and $C>0$ such that $\mathfrak{L}(\lambda)$ is an isomorphism whenever $0<\left|\lambda-\lambda_{0}\right|<\varepsilon$, and

$$
\left\|\mathfrak{L}^{-1}(\lambda)\right\|<\frac{C}{\left|\lambda-\lambda_{0}\right|^{\kappa}} \quad \text { if } 0<\left|\lambda-\lambda_{0}\right|<\varepsilon,
$$

with $\kappa$ the minimal integer for which (1.2) holds. Throughout this paper, the set of $\kappa$ transversal eigenvalues of $\mathfrak{L}(\lambda)$ is denoted by $\operatorname{Alg}_{\kappa}(\mathfrak{L})$, and the set of algebraic eigenvalues of $\mathfrak{L}$ is defined by

$$
\operatorname{Alg}(\mathfrak{L}):=\bigcup_{\kappa \in \mathbb{N}} \operatorname{Alg}_{\kappa}(\mathfrak{L})
$$

It turns out that the multiplicities of [25, 7, 6] are well defined if, and only if, the path $\mathfrak{L}(\lambda)$ is of class $\mathcal{C}^{r}$ for some $r \geq 1$ and $\lambda_{0} \in \operatorname{Alg}_{\kappa}(\mathfrak{L})$ for some $1 \leq \kappa \leq r$. Moreover, by Theorems 4.4.1 and 4.4.4 of López-Gómez [20], when $\mathfrak{L}$ is analytic and $\mathfrak{L}(\Omega)$ contains some invertible operator, then $\Sigma(\mathfrak{L})$ is discrete, and every $\lambda_{0} \in \Sigma(\mathfrak{L})$ is an algebraic eigenvalue of $\mathfrak{L}$. Thus, through this paper, it is convenient to denote by $\mathcal{A}_{\lambda_{0}}\left(\Omega_{\lambda_{0}}, \Phi_{0}(X, Y)\right)$ the set of curves $\mathfrak{L} \in \mathcal{C}^{r}\left(\Omega_{\lambda_{0}}, \Phi_{0}(X, Y)\right)$ such that $\lambda_{0} \in \operatorname{Alg}_{\kappa}(\mathfrak{L}) \cap \Omega_{\lambda_{0}}$ with $1 \leq \kappa \leq r$ for some $r \in \mathbb{N}$. According to [20, Ch. 4], the generalized algebraic multiplicity $\chi\left[\mathfrak{L}, \lambda_{0}\right]$ is well defined if and only if $\mathfrak{L} \in \mathcal{A}_{\lambda_{0}}\left(\Omega_{\lambda_{0}}, \Phi_{0}(X, Y)\right)$.

Short time later, in 2004, the theory of algebraic multiplicities for $\mathcal{C}^{\infty}$-Fredholm paths was axiomatized by Mora-Corral [26] by establishing that, modulus a normalization condition (see Theorem 2.2 for the precise statement), given an open connected neighborhood of $\lambda_{0}$ in $\mathbb{K}$, denoted by $\Omega_{\lambda_{0}}$, the algebraic multiplicity $\chi$ is the unique map

$$
\chi\left[\cdot, \lambda_{0}\right]: \mathcal{C}^{\infty}\left(\Omega_{\lambda_{0}}, \Phi_{0}(U)\right) \longrightarrow[0, \infty]
$$

satisfying the product formula

$$
\chi\left[\mathfrak{L} \circ \mathfrak{M}, \lambda_{0}\right]=\chi\left[\mathfrak{L}, \lambda_{0}\right]+\chi\left[\mathfrak{M}, \lambda_{0}\right]
$$

for all $\mathfrak{L}, \mathfrak{M} \in \mathcal{C}^{\infty}\left(\Omega_{\lambda_{0}}, \Phi_{0}(U)\right)$, regardless whether, or not, $\lambda_{0}$ is singular. These findings were collected by López-Gómez and Mora-Corral in [23], where, in addition, the theory of Göhberg and Sigal [11] was substantially generalized to a non-holomorphic setting, and the existence of the local Smith form was established in the class $\mathcal{A}_{\lambda_{0}}\left(\Omega_{\lambda_{0}}, \Phi_{0}(X, Y)\right)$ through the lengths of the Jordan chains of $\mathfrak{L}(\lambda)$. 
The relevance of $\chi\left[\mathfrak{L}, \lambda_{0}\right]$ in Nonlinear Analysis relies on the crucial fact that, in the special case when $U=V, \mathbb{K}=\mathbb{R}, \Omega_{\lambda_{0}}=(a, b)$ for some $a<b, \lambda_{0} \in(a, b)$ is isolated in $\Sigma(\mathfrak{L})$, and $I_{U}-\mathfrak{L}(\lambda)$ is compact for all $\lambda \in(a, b)$, by [20, Th. 5.6.2], $\chi\left[\mathfrak{L}, \lambda_{0}\right]$ is odd if and only if the Leray-Schauder degree $\operatorname{deg}_{L S}\left(\mathfrak{L}(\lambda), B_{R}(0)\right)$ changes as $\lambda$ crosses $\lambda_{0}$ (see López-Gómez and Sampedro [24] for far more general results in the Fredholm context). In this paper, for any given $u_{0} \in U$ and $R>0$, we denote by $B_{R}\left(u_{0}\right)$ the ball of radius $R$ centered at $u_{0}$. Note that, whenever $\operatorname{dim} U<+\infty$, one can chose a basis in $U$ so that

$$
\operatorname{deg}_{L S}\left(\mathfrak{L}(\lambda), B_{R}(0)\right)=\operatorname{sign} \operatorname{det} \mathfrak{L}(\lambda)
$$

for all $\lambda \in(a, b) \backslash \Sigma(\mathfrak{L})$. These changes of degree have relevant consequences in Nonlinear Analysis, because they entail that, for every completely continuous map $\mathfrak{N}(\lambda, u)$ such that $\mathfrak{N}(\lambda, 0)=0$ and $\mathfrak{N}(\lambda, u)=o(\|u\|)$ as $u \rightarrow 0$, the set of nontrivial solutions of the equation

$$
\mathscr{F}(\lambda, u) \equiv \mathfrak{L}(\lambda) u+\mathfrak{N}(\lambda, u)=0
$$

possesses a connected component, denoted by $\mathscr{C}$, bifurcating from $u=0$ at $\lambda=\lambda_{0}$ (see [20, 21]). By a nontrivial solution it is meant any pair $(\lambda, u) \in \mathscr{F}^{-1}(0)$ with $u \neq 0$. Surprisingly, this occurs for every completely continuous nonlinearity $\mathfrak{N}(\lambda, u)$ if and only if $\chi\left[\mathfrak{L}, \lambda_{0}\right]$ is odd. And, in such case, the component $\mathscr{C}$ satisfies the global alternative of Rabinowitz [29], as well as the global theorems of Nirenberg [27] and Magnus [25] (see [20, Th. 6.3.1]). Indeed, when $(a, b)=\mathbb{R}$, either

(a) $\mathscr{C}$ is unbounded in $\mathbb{R} \times U$; or

(b) there exists $\lambda_{1} \neq \lambda_{0}$ such that $\left(\lambda_{1}, 0\right) \in \overline{\mathscr{C}}$.

The Leray-Schauder degree had been incorporated to the weaponry of Nonlinear Integral Equations by Krasnoselskij [16] in order to prove his celebrated local bifurcation theorem, establishing that if $K: U \rightarrow U$ is compact, $\mathfrak{L}(\lambda)=\lambda I_{U}-K$ for all $\lambda \in(a, b)$, and $\lambda_{0} \in$ $\sigma(K)$ is an eigenvalue with odd (classical) algebraic multiplicity, then the component $\mathscr{C}$ exists for every completely continuous map $\mathfrak{N}(\lambda, u)$ satisfying the previous requirements.

The first aim of this paper is to identify $\chi\left[\mathfrak{L}, \lambda_{0}\right]$ in terms of the Schur operator of the path $\mathfrak{L}(\lambda)$. This provides us with a definition of $\chi$ as a natural extension of the finite dimensional concept (1.1) adopting a direct approach. In this paper, for any given $T \in \Phi_{0}(U, V)$, we will denote by $N[T]$ the null space, or kernel, of $T$, and by $R[T]$ the range, or image, of $T$. Assume $\lambda_{0} \in \Sigma(\mathfrak{L})$ and let $P \in \mathcal{L}(U)$ and $Q \in \mathcal{L}(V)$ be a pair of projections onto $N\left[\mathfrak{L}\left(\lambda_{0}\right)\right]$ and $R\left[\mathfrak{L}\left(\lambda_{0}\right)\right]$, respectively. Then,

$$
U=\left(I_{U}-P\right)(U) \oplus N\left[\mathfrak{L}\left(\lambda_{0}\right)\right], \quad V=R\left[\mathfrak{L}\left(\lambda_{0}\right)\right] \oplus\left(I_{V}-Q\right)(V),
$$

and $\mathfrak{L}(\lambda)$ can be expressed as a block operator matrix

$$
\mathfrak{L}(\lambda)=\left(\begin{array}{cc}
L_{11}(\lambda) & L_{12}(\lambda) \\
L_{21}(\lambda) & L_{22}(\lambda)
\end{array}\right)
$$

where

$$
\begin{array}{ll}
L_{11}(\lambda):=Q \mathfrak{L}(\lambda)\left(I_{U}-P\right), & L_{12}(\lambda):=Q \mathfrak{L}(\lambda) P, \\
L_{21}(\lambda):=\left(I_{V}-Q\right) \mathfrak{L}(\lambda)\left(I_{U}-P\right), & L_{22}(\lambda):=\left(I_{V}-Q\right) \mathfrak{L}(\lambda) P .
\end{array}
$$

In this context, the Schur operator of $\mathfrak{L}(\lambda)$ associated to the projection pair $(P, Q)$ can be defined through

$$
\mathscr{S}_{\mathfrak{L}(\lambda),(P, Q)}(\mathfrak{L}(\lambda)):=L_{22}(\lambda)-L_{21}(\lambda) L_{11}^{-1}(\lambda) L_{12}(\lambda), \quad \lambda \in \Omega_{\lambda_{0}} .
$$

In terms of this operator, our first result can be stated as follows. 
Theorem 1.1. Assume $\mathfrak{L} \in \mathcal{C}\left(\Omega, \Phi_{0}(U, V)\right)$ and $\lambda_{0} \in \Omega \cap \Sigma(\mathfrak{L})$ is an isolated eigenvalue. Then, for every pair $(P, Q)$ of $\mathfrak{L}\left(\lambda_{0}\right)$-projections,

$$
\mathscr{S}_{\mathfrak{L}\left(\lambda_{0}\right),(P, Q)}^{-1}(\mathfrak{L}(\lambda))=P \mathfrak{L}^{-1}(\lambda)\left(I_{V}-Q\right) .
$$

If, in addition, $\mathfrak{L} \in \mathcal{A}_{\lambda_{0}}\left(\Omega_{\lambda_{0}}, \Phi_{0}(X, Y)\right)$, then

$$
\chi\left[\mathfrak{L}, \lambda_{0}\right]=\operatorname{ord}_{\lambda_{0}} \operatorname{det} \mathscr{S}_{\mathfrak{L}\left(\lambda_{0}\right),(P, Q)}(\mathfrak{L}(\lambda))=\operatorname{ord}_{\lambda_{0}} \operatorname{det}\left[P \mathfrak{L}^{-1}(\lambda)\left(I_{V}-Q\right)\right]^{-1} .
$$

Theorem 1.1 allows us to introduce, in a rather natural manner, a local concept of infinite-dimensional determinant, $\mathcal{D}_{\mathfrak{L}\left(\lambda_{0}\right),(P, Q)}$, for which

$$
\chi\left[\mathfrak{L}, \lambda_{0}\right]=\operatorname{ord}_{\lambda_{0}} \mathcal{D}_{\mathfrak{L}\left(\lambda_{0}\right),(P, Q)}(\mathfrak{L}(\lambda))=\operatorname{ord}_{\lambda_{0}} \operatorname{det} \mathscr{S}_{\mathfrak{L}\left(\lambda_{0}\right),(P, Q)}(\mathfrak{L}(\lambda)) .
$$

Indeed, we will see that taking

$$
\mathcal{D}_{\mathfrak{L}\left(\lambda_{0}\right),(P, Q)}(\mathfrak{L}(\lambda)):=\operatorname{det}\left(L_{11}(\lambda)\right) \cdot \operatorname{det}\left(\mathscr{S}_{T,(P, Q)}(\mathfrak{L}(\lambda))\right.
$$

is consistent with the classical theory of Schur complements. As a byproduct of Theorem 1.1. the multiplicity of Ize [15], introduced for analytic paths, can be defined mutatis mutandis for every admissible path $\mathfrak{L} \in \mathcal{A}_{\lambda_{0}}\left(\Omega_{\lambda_{0}}, \Phi_{0}(X, Y)\right)$. Our next result establishes a sharp connection between these concepts and Bifurcation Theory.

Theorem 1.2. Assume $\mathfrak{L} \in \mathcal{C}^{1}\left(\Omega, \Phi_{0}(U, V)\right)$ and $\lambda_{0} \in \Omega$. Then, for every pair $(P, Q)$ of $\mathfrak{L}\left(\lambda_{0}\right)$-projections, the Schur operator $\mathscr{S}_{\mathfrak{L}\left(\lambda_{0}\right),(P, Q)}(\mathfrak{L}(\lambda))$ coincides with the linearization at $(\lambda, u)=(\lambda, 0)$ of the bifurcation equation of (1.3) through the Lyapunov-Schmidt decomposition of projections $(P, Q)$, derived as discussed in [20, Ch. 3], which is denoted by $\mathscr{B}(\lambda)$ in this paper (see Section 4), i.e.

$$
\mathscr{B}(\lambda)=\mathscr{S}_{\mathfrak{L}\left(\lambda_{0}\right),(P, Q)}(\mathfrak{L}(\lambda))=L_{22}(\lambda)-L_{21}(\lambda) L_{11}^{-1}(\lambda) L_{12}(\lambda) .
$$

If, in addition, $\mathfrak{L} \in \mathcal{A}_{\lambda_{0}}\left(\Omega_{\lambda_{0}}, \Phi_{0}(X, Y)\right)$, then, thanks to Theorem 1.1,

$$
\chi\left[\mathfrak{L}, \lambda_{0}\right]=\operatorname{ord}_{\lambda_{0}} \operatorname{det} \mathscr{S}_{\mathfrak{L}\left(\lambda_{0}\right),(P, Q)}(\mathfrak{L}(\lambda))=\operatorname{ord}_{\lambda_{0}} \operatorname{det} \mathscr{B}(\lambda),
$$

regardless the pair of projections chosen, $(P, Q)$. Therefore, thanks to Theorem $[20$, Th. 4.3.4], the following assertions are equivalent:

(a) $\lambda_{0}$ is a nonlinear eigenvalue of $\mathfrak{L}(\lambda)$, in the sense that $\left(\lambda_{0}, 0\right)$ is a bifurcation point of (1.3) from $(\lambda, u)=(\lambda, 0)$ for all map $\mathfrak{N}(\lambda, u)$ of class $\mathcal{C}^{1}$.

(b) $\chi\left[\mathfrak{L}, \lambda_{0}\right]$ is an odd integer.

(c) $\operatorname{det} \mathscr{B}(\lambda)$ changes of sign as $\lambda$ crosses $\lambda_{0}$, regardless $(P, Q)$.

(d) $\operatorname{det} \mathscr{S}_{\mathfrak{L}(\lambda),(P, Q)}(\mathfrak{L}(\lambda))$ changes of sign as $\lambda$ crosses $\lambda_{0}$, regardless $(P, Q)$.

(e) $\operatorname{det}\left[P \mathfrak{L}^{-1}(\lambda)\left(I_{V}-Q\right)\right]$ changes of sign as $\lambda$ crosses $\lambda_{0}$, regardless $(P, Q)$.

Furthermore, when any of these conditions occurs, there is a component, $\mathscr{C}$, of the set of nontrivial solutions of (4.1) such that $\left(\lambda_{0}, 0\right) \in \overline{\mathscr{C}}$. By a component, it is meant any closed and connected subset maximal for the inclusion.

Our second aim is incorporating a completely new perspective into the theory of algebraic multiplicities in order to establish a bisociation between Spectral Theory and Algebraic Geometry. As already discussed above, any generalized eigenvalue $\lambda_{0} \in \Sigma(\mathfrak{L})$ of the curve $\mathfrak{L} \in \mathcal{C}\left(\Omega, \Phi_{0}(U, V)\right)$ lies in the intersection of $\mathfrak{L}$ with the singular space $\mathcal{S}(U, V) \cap \Phi_{0}(U, V)$. This geometrical feature leads us to think that the algebraic multiplicity might actually measure how the curve $\mathfrak{L}(\lambda)$ intersects geometrically with $\mathcal{S}(U, V) \cap$ $\Phi_{0}(U, V)$. Thus, it might be related to the local intersection index, a pivotal geometrical device for measuring the nature of the intersections of varieties.

Given a family of algebraic varieties, $\mathscr{V}$, an intersection theory over $\mathscr{V}$ consists of giving a pairing

$$
\text { - : } A^{r}(X) \times A^{s}(X) \rightarrow A^{r+s}(X)
$$


satisfying a series of axioms (see, e.g., Hartshorne [14, pp. 426-427], Eisenbud and Harris [5, Ch. $1 \& 2$ ], and Fulton [13, Ch. $7 \& 8])$ for every $r, s \in \mathbb{N}$ and $X \in \mathscr{V}$, where $A^{r}(X)$ stands for the group of cycles of codimension $r$ on $X$ modulo rational equivalence. The graded group

$$
A(X) \equiv \bigoplus_{r \in \mathbb{N}} A^{r}(X)
$$

is referred to as the Chow group after Chow [3]. The pairing (1.11) gives to $A(X)$ the structure of a graded ring, the Chow ring of $X$. Giving an intersection theory to an algebraic variety $X$ consists in giving the structure of the Chow ring $A(X)$; the axioms of the pairing $\bullet$ try to mimic in $X$ the celebrated Bezout Theorem [5, \& 2.1.1]. This explains why one of these axioms establishes that if $X_{1}$ and $X_{2}$ are subvarieties of $X$ with proper intersection, in the sense that any irreducible component of $X_{1} \cap X_{2}$ has codimension $\operatorname{codim} X_{1}+\operatorname{codim} X_{2}$, then

$$
\left[X_{1}\right] \bullet\left[X_{2}\right]=\sum_{j} i\left(X_{1}, X_{2} ; C_{j}\right)\left[C_{j}\right]
$$

where the sum runs over the set of all irreducible components, $C_{j}$, of $X_{1} \cap X_{2}$, and the integer $i\left(X_{1}, X_{2} ; C_{j}\right)$ stands for the local intersection index of $X_{1}$ and $X_{2}$ along $C_{j}$. By a result of Serre [30, Ch. V, \& C.1], for any given pair $X_{1}, X_{2}$ of subvarieties of a smooth variety $X$ with proper intersection and any irreducible component, $C$, of $X_{1} \cap X_{2}$, the local intersection index of $X_{1}$ and $X_{2}$ along $C$ is given through

$$
i\left(X_{1}, X_{2} ; C\right)=\sum_{i=0}^{\operatorname{dim} X}(-1)^{i} \ell_{\mathcal{O}_{c, X}} \operatorname{Tor}_{i}^{\mathcal{O}_{c, X}}\left(\mathcal{O}_{c, X} / \mathfrak{P}_{X_{1}}, \mathcal{O}_{c, X} / \mathfrak{P}_{X_{2}}\right)
$$

where $\mathcal{O}_{c, X}$ is the local ring of $c \in C$ in $X$, and $\mathfrak{P}_{X_{1}}$ and $\mathfrak{P}_{X_{2}}$ are the ideals of $X_{1}$ and $X_{2}$, respectively, in the ring $\mathcal{O}_{c, X}$. In this paper, and, in particular, in the Serre formula, we are denoting by $\ell_{R}(M)$ the length of the module $M$ over the $\operatorname{ring} R$, and by $\operatorname{Tor}_{i}^{R}$ the $i$-th Tor $R$-module. As in our context $X$ is a smooth algebraic variety over an algebraically closed field, $\mathbb{C}$, and $X_{1}, X_{2}$ are two irreducible Cohen-Macaulay subvarieties of $X$ such that $X_{1} \cap X_{2}=\{x\}$, with proper intersection, according to, e.g., Eisenbaud and Harris [5. p. 48], the local intersection index of $X_{1}$ and $X_{2}$ at $x$ reduces to

$$
i\left(X_{1}, X_{2} ; x\right):=\ell_{\mathcal{O}_{x, X}}\left(\mathcal{O}_{x, X} /\left(\mathfrak{P}_{X_{1}}+\mathfrak{P}_{X_{2}}\right)\right) .
$$

The third finding of this paper establishes a connection between the concepts of classical algebraic multiplicity and the intersection index. Subsequently, $\mathcal{D}: \mathcal{L}\left(\mathbb{C}^{N}\right) \rightarrow \mathbb{C}$ stands for the determinant map defined by

$$
\mathcal{D}(T):=\operatorname{det} T \text { for every } T \in \mathcal{L}\left(\mathbb{C}^{N}\right) .
$$

Theorem 1.3. Let $T \in \mathcal{L}\left(\mathbb{C}^{N}\right)$ and $\lambda_{0} \in \sigma(T)$ be, and set $\mathfrak{L}(\lambda)=\lambda I_{N}-T, \lambda \in \mathbb{C}$. Then,

$$
\chi\left[\mathfrak{L}, \lambda_{0}\right]=i\left(\mathcal{D}^{-1}(0), \mathfrak{L}(\mathbb{C}) ; \mathfrak{L}\left(\lambda_{0}\right)\right) .
$$

Finally, in order to state our last finding, for any given $\mathfrak{L} \in \mathcal{A}_{\lambda_{0}}\left(\Omega_{\lambda_{0}}, \Phi_{0}(X, Y)\right)$, we will denote by

$$
\mathscr{L}=\mathscr{L}(\mathfrak{L}) \in \mathcal{L}\left(\mathbb{C}^{M}\right)
$$

the global linearization, as discussed by Lemma 10.1.1 of [23], of the local Smith form of the Schur complement of $\mathfrak{L}(\lambda)$, whose existence follows from Theorem 7.4.1 of López-Gómez and Mora-Corral [23]. The following result is a substantial, very sharp, generalization of Theorem 1.3 . 
Theorem 1.4. For every $\mathfrak{L} \in \mathcal{A}_{\lambda_{0}}\left(\Omega_{\lambda_{0}}, \Phi_{0}(X, Y)\right)$,

$$
\chi\left[\mathfrak{L}, \lambda_{0}\right]=i\left(\mathcal{D}^{-1}(0), \lambda I_{M}-\mathscr{L} ; \lambda_{0} I_{M}-\mathscr{L}\right) .
$$

This paper is distributed as follows. Section 2 reviews briefly the generalized algebraic multiplicity $\chi\left[\mathfrak{L}, \lambda_{0}\right]$. In Section 3 we introduce a local notion of determinant for a general path $\mathfrak{L} \in \mathcal{C}\left(\Omega, \Phi_{0}(U, V)\right)$ through the Schur operator $\mathscr{S}_{\mathfrak{L}(\lambda),(P, Q)}(\mathfrak{L}(\lambda))$ associated to a projection pair $(P, Q)$. Then, we use this concept of infinite-dimensional determinant to prove Theorem 1.1. Section 4 consists of the proof of Theorem 1.2, Section 5 shows Theorem 1.3 and, finally, Section 6 proves Theorem 1.4. In reading this paper, it would be appropriate to have copies of [20] and [23].

\section{Nonlinear speCtral theory. Generalized algebraic multiplicity.}

Throughout this section, $\mathfrak{L} \in \mathcal{C}\left(\Omega, \Phi_{0}(U, V)\right)$, where $\Omega$ is a subdomain of $\mathbb{K} \in\{\mathbb{R}, \mathbb{C}\}$. According to Theorems 4.4 .1 and 4.4 .4 of [20], when $\mathfrak{L}(\lambda)$ is analytic in $\Omega$, i.e., $\mathfrak{L} \in$ $\mathcal{H}\left(\Omega, \Phi_{0}(U, V)\right)$, either $\Sigma(\mathfrak{L})=\Omega$, or $\Sigma(\mathfrak{L})$ is discrete and $\Sigma(\mathfrak{L}) \subset \operatorname{Alg}(\mathfrak{L})$.

The next concept, going back to Esquinas and López-Gómez [7], is pivotal in Nonlinear Spectral Theory as it allows to introduce a generalized algebraic multiplicity, $\chi\left[\mathfrak{L}, \lambda_{0}\right]$, in a rather natural manner. Subsequently, we will denote

$$
\mathfrak{L}_{j}:=\frac{1}{j !} \mathfrak{L}^{(j)}\left(\lambda_{0}\right), \quad 0 \leq j \leq r
$$

if these derivatives exist.

Definition 2.1. Let $\mathfrak{L} \in \mathcal{C}^{r}\left(\Omega, \Phi_{0}(U, V)\right)$ and $1 \leq \kappa \leq r$. Then, a given $\lambda_{0} \in \Sigma(\mathfrak{L})$ is said to be a $\kappa$-transversal eigenvalue of $\mathfrak{L}$ if

$$
\bigoplus_{j=1}^{\kappa} \mathfrak{L}_{j}\left(\bigcap_{i=0}^{j-1} N\left[\mathfrak{L}_{i}\right]\right) \oplus R\left(\mathfrak{L}_{0}\right)=V \text { with } \mathfrak{L}_{\kappa}\left(\bigcap_{i=0}^{\kappa-1} N\left[\mathfrak{L}_{i}\right]\right) \neq\{0\} .
$$

For these eigenvalues, the algebraic multiplicity of $\mathfrak{L}$ at $\lambda_{0}, \chi\left[\mathfrak{L}, \lambda_{0}\right]$, was introduced in [7] through

$$
\chi\left[\mathfrak{L}, \lambda_{0}\right]:=\sum_{j=1}^{\kappa} j \cdot \operatorname{dim} \mathfrak{L}_{j}\left(\bigcap_{i=0}^{j-1} N\left[\mathfrak{L}_{i}\right]\right) .
$$

According to Theorems 4.3.2 and 5.3.3 of [20], for every $\mathfrak{L} \in \mathcal{A}_{\lambda_{0}}\left(\Omega, \Phi_{0}(U, V)\right)$ there exists a polynomial $\Phi: \mathbb{K} \rightarrow \mathcal{L}(U)$ with $\Phi\left(\lambda_{0}\right)=I_{U}$ and an integer $\kappa \in\{1,2, \ldots, r\}$ such that $\lambda_{0}$ is a $\kappa$-transversal eigenvalue of the path

$$
\mathfrak{L}^{\Phi}:=\mathfrak{L} \circ \Phi \in \mathcal{C}^{r}\left(\Omega, \Phi_{0}(U, V)\right) .
$$

Moreover, $\kappa$ and $\chi\left[\mathfrak{L}^{\Phi}, \lambda_{0}\right]$ are independent of the curve of trasversalizing local isomorphisms $\Phi$ chosen to transversalize $\mathfrak{L}$ at $\lambda_{0}$ through (2.1), regardless $\Phi$ is a polynomial or not. Therefore, the next generalized concept of algebraic multiplicity, defined for every $\mathfrak{L} \in \mathcal{A}_{\lambda_{0}}\left(\Omega, \Phi_{0}(U, V)\right)$, is consistent

$$
\chi\left[\mathfrak{L}, \lambda_{0}\right]:=\chi\left[\mathfrak{L}^{\Phi}, \lambda_{0}\right] .
$$

This concept can be extended by setting

$$
\chi\left[\mathfrak{L}, \lambda_{0}\right]:=0 \quad \text { if } \quad \lambda_{0} \notin \Sigma(\mathfrak{L})
$$

and

$$
\chi\left[\mathfrak{L}, \lambda_{0}\right]:=+\infty \quad \text { if } \lambda_{0} \in \Sigma(\mathfrak{L}) \backslash \operatorname{Alg}(\mathfrak{L}) \text { and } r=+\infty
$$


so that $\chi[\mathfrak{L}, \lambda]$ is well defined for all $\mathfrak{L} \in \mathcal{A}_{\lambda_{0}}\left(\Omega, \Phi_{0}(U, V)\right)$ at any $\lambda \in \Omega$, and, in particular, for every analytic curve $\mathfrak{L} \in \mathcal{H}\left(\Omega, \Phi_{0}(U, V)\right)$. Setting (2.2), the algebraic multiplicity is also well defined for each smooth curve $\mathfrak{L} \in \mathcal{C}^{\infty}\left(\Omega, \Phi_{0}(U, V)\right)$ at any $\lambda \in \Omega$, and it coincides with the multiplicity of Magnus [25]. The next uniqueness result, going back to Mora-Corral [26], axiomatizes the concept of Algebraic Multiplicity (see also Chapter 6 of [23]).

Theorem 2.2. For every $\lambda_{0} \in \mathbb{K}$ and any open neighborhood $\Omega_{\lambda_{0}} \subset \mathbb{K}$ of $\lambda_{0}$, the algebraic multiplicity $\chi$ is the unique map

$$
\chi\left[\cdot, \lambda_{0}\right]: \mathcal{C}^{\infty}\left(\Omega_{\lambda_{0}}, \Phi_{0}(U)\right) \longrightarrow[0, \infty]
$$

such that

(PF) For every pair $\mathfrak{L}, \mathfrak{M} \in \mathcal{C}^{\infty}\left(\Omega_{\lambda_{0}}, \Phi_{0}(U)\right)$,

$$
\chi\left[\mathfrak{L} \circ \mathfrak{M}, \lambda_{0}\right]=\chi\left[\mathfrak{L}, \lambda_{0}\right]+\chi\left[\mathfrak{M}, \lambda_{0}\right] .
$$

(NP) There exists a rank one projection $\Pi \in \mathcal{L}(U)$ such that

$$
\chi\left[\left(\lambda-\lambda_{0}\right) \Pi+I_{U}-\Pi, \lambda_{0}\right]=1 .
$$

The axiom (PF) is the product formula and (NP) is a normalization property for establishing the uniqueness of $\chi$. From these two axioms one can derive the remaining properties of $\chi$; among them, that it equals the classical algebraic multiplicity when

$$
\mathfrak{L}(\lambda)=\lambda I_{U}-K
$$

for some compact operator $K$. Indeed, according to [23], for every $\mathfrak{L} \in \mathcal{C}^{\infty}\left(\Omega_{\lambda_{0}}, \Phi_{0}(U)\right)$, the following properties are satisfied:

- $\chi\left[\mathfrak{L}, \lambda_{0}\right] \in \mathbb{N} \uplus\{+\infty\}$;

- $\chi\left[\mathfrak{L}, \lambda_{0}\right]=0$ if and only if $\mathfrak{L}\left(\lambda_{0}\right) \in G L(U)$;

- $\chi\left[\mathfrak{L}, \lambda_{0}\right]<\infty$ if and only if $\lambda_{0} \in \operatorname{Alg}(\mathfrak{L})$.

- If $U=\mathbb{K}^{N}$, then, in any basis,

$$
\chi\left[\mathfrak{L}, \lambda_{0}\right]=\operatorname{ord}_{\lambda_{0}} \operatorname{det} \mathfrak{L}(\lambda) .
$$

- Let $L \in \mathcal{L}(U)$ be such that $\lambda I_{U}-L \in \Phi_{0}(U)$. Then, for each eigenvalue $\lambda_{0} \in \sigma(L)$, there exists $\kappa \geq 1$ such that

$$
\chi\left[\lambda I_{U}-L, \lambda_{0}\right]=\sup _{n \in \mathbb{N}} \operatorname{dim} N\left[\left(\lambda_{0} I_{U}-L\right)^{n}\right]=\operatorname{dim} N\left[\left(\lambda_{0} I_{U}-L\right)^{\kappa}\right] .
$$

- Let $V$ be another complex Banach space and $\mathfrak{P} \in \mathcal{C}^{\infty}\left(\Omega_{\lambda_{0}}, \Phi_{0}(V)\right)$. Then,

$$
\chi\left[\mathfrak{L} \oplus \mathfrak{P}, \lambda_{0}\right]=\chi\left[\mathfrak{L}, \lambda_{0}\right]+\chi\left[\mathfrak{P}, \lambda_{0}\right] .
$$

Therefore, $\chi$ extends, very substantially, the classical concept of algebraic multiplicity, which is only valid for operator paths of the form (2.3).

\section{SCHUR COMPlEMENT AND LOCAL DETERMinANT}

This section gives a new equivalent definition of $\chi$, without invoking the concept of transversalization, based on the formulation of the finite-dimensional algebraic multiplicity in terms of the order of the determinant. To accomplish this task, we need to develop an analogue of the notion of determinant in an infinite-dimensional setting. Although the notion of determinant does not exists in such setting, we will see that it can be introduced in local neighborhoods of $\Phi_{0}(U, V)$.

Throughout this section, $T \in \Phi_{0}(U, V)$ is an arbitrary linear Fredholm operator of index zero, and $P \in \mathcal{L}(U), Q \in \mathcal{L}(V)$ are projections onto $N[T]$ and $R[T]$, respectively; 
The pair $(P, Q)$ will be called a pair of $T$-projections. Then, the following topological direct sum decompositions hold

$$
U=\left(I_{U}-P\right)(U) \oplus N[T], \quad V=R[T] \oplus\left(I_{V}-Q\right)(V),
$$

and, setting

$$
\begin{aligned}
& R\left[I_{U}-P\right]=\left(I_{U}-P\right)(U) \equiv N[T]^{\perp}, \\
& R\left[I_{V}-Q\right]=\left(I_{V}-Q\right)(V) \equiv R[T]^{\perp},
\end{aligned}
$$

by Fitzpatrick and Pejsachowicz [12, p. 286], for any given $L \in \Phi_{0}(U, V), L$ can be equivalently expressed as a block operator matrix

$$
L=\left(\begin{array}{ll}
L_{11} & L_{12} \\
L_{21} & L_{22}
\end{array}\right)
$$

where

$$
\begin{array}{ll}
L_{11}:=Q L\left(I_{U}-P\right), & L_{12}:=Q L P, \\
L_{21}:=\left(I_{V}-Q\right) L\left(I_{U}-P\right), & L_{22}:=\left(I_{V}-Q\right) L P .
\end{array}
$$

In particular, since $T P=0$ and $\left(I_{V}-Q\right) T=0$, the operator $T$ can be expressed as

$$
T=\left(\begin{array}{cc}
T_{11} & 0 \\
0 & 0
\end{array}\right)
$$

with $T_{11} \in G L\left(N[T]^{\perp}, R[T]\right)$. Since $G L\left(N[T]^{\perp}, R[T]\right)$ is open in $\mathcal{L}\left(N[T]^{\perp}, R[T]\right)$ and $\Phi_{0}(U, V)$ is open in $\mathcal{L}(U, V)$, there exists $\varepsilon>0$ such that, whenever $L \in \mathcal{L}(U, V)$ satisfies $\|T-L\|<\varepsilon$, then $L \in \Phi_{0}(U, V)$, and it can be expressed as (3.2) with $L_{11} \in G L\left(N[T]^{\perp}, R[T]\right)$.

In this context, the Schur operator of $T$ associated to the projection pair $(P, Q)$ can be defined through

$$
\begin{array}{cl}
\mathscr{S}_{T,(P, Q)}: B_{\varepsilon}(T) \subset \Phi_{0}(U, V) & \longrightarrow \mathcal{L}\left(N[T], R[T]^{\perp}\right) \\
L & \mapsto L_{22}-L_{21} L_{11}^{-1} L_{12}
\end{array}
$$

where $B_{\varepsilon}(T)$ stands for the open ball of radius $\varepsilon$ centered at $T \in \Phi_{0}(U, V)$ in $\mathcal{L}(U, V)$. We call this map Schur operator by analogy, in the Euclidean space, to the Schur complement of a matrix. Indeed, given any block matrix

$$
M=\left(\begin{array}{cc}
A & B \\
C & D
\end{array}\right)
$$

with $A \in G L\left(\mathbb{K}^{n}\right), B \in \operatorname{Mat}_{n \times m}(\mathbb{K}), C \in \operatorname{Mat}_{m \times n}(\mathbb{K})$ and $D \in \operatorname{Mat}_{m}(\mathbb{K})$, the Schur complement of the matrix $D$ in $M$ is the matrix $M / A \in \operatorname{Mat}_{m}(\mathbb{K})$ defined by

$$
M / A:=D-C A^{-1} B .
$$

By a lemma of Banachiewicz [1, p. 50], the Schur complement satisfies the identity

$$
\operatorname{det}(M)=\operatorname{det}(A) \cdot \operatorname{det}(M / A)
$$

for every block matrix (3.3). Based on this feature, we introduce the following local notion of determinant.

Definition 3.1. Let $T \in \Phi_{0}(U, V)$ and $(P, Q)$ be a pair of $T$-projections. Then, for sufficiently small $\varepsilon>0$, we define the local determinant functional by

$$
\begin{aligned}
\mathcal{D}_{T,(P, Q)}: B_{\varepsilon}(T) \subset \Phi_{0}(U, V) & \longrightarrow \mathbb{K} \\
L & \mapsto \operatorname{det}\left(L_{11}\right) \cdot \operatorname{det}\left(\mathscr{S}_{T,(P, Q)}(L)\right) .
\end{aligned}
$$


The next result establishes that the local determinant functional indeed behaves as a (local) determinant on $B_{\varepsilon}(T)$.

Theorem 3.2. Let $T \in \Phi_{0}(U, V)$ and $(P, Q)$ be a pair of $T$-projections. Then, for sufficiently small $\epsilon>0$ and every $L \in B_{\varepsilon}(T)$,

$$
L \in G L(U, V) \text { if and only if } \mathcal{D}_{T,(P, Q)}(L) \neq 0 \text {. }
$$

Proof. Let $L \in B_{\varepsilon}(T)$ be. Then, the Schur operator of $L$ is well defined. Moreover, $L$ can be decomposed as

$$
L=\left(\begin{array}{cc}
I_{R[T]} & 0 \\
L_{21} L_{11}^{-1} & I_{R[T]^{\perp}}
\end{array}\right)\left(\begin{array}{cc}
L_{11} & 0 \\
0 & \mathscr{S}_{T,(P, Q)}(L)
\end{array}\right)\left(\begin{array}{cc}
I_{N[T]^{\perp}} & L_{11}^{-1} L_{12} \\
0 & I_{N[T]}
\end{array}\right) .
$$

Clearly, the first and the third matrix operators of (3.4) are invertible. Thus, taking inverses, we find that

$$
\begin{aligned}
L_{11} \oplus \mathscr{S}_{T,(P, Q)}(L) & =\left(\begin{array}{cc}
L_{11} & 0 \\
0 & \mathscr{S}_{T,(P, Q)}(L)
\end{array}\right) \\
& =\left(\begin{array}{cc}
I_{R[T]} & 0 \\
L_{21} L_{11}^{-1} & I_{R[T]^{\perp}}
\end{array}\right)^{-1} L\left(\begin{array}{cc}
I_{N[T]^{\perp}} & L_{11}^{-1} L_{12} \\
0 & I_{N[T]}
\end{array}\right)^{-1} .
\end{aligned}
$$

If $L \in G L(U, V)$, then, due to (3.5), $L_{11} \oplus \mathscr{S}_{T,(P, Q)} \in G L(U, V)$ because it is a composition of invertible operators. So, since $L_{11} \in G L\left(N[T]^{\perp}, R[T]\right)$, necessarily

$$
\mathscr{S}_{T,(P, Q)}(L) \in G L\left(N[T], R[T]^{\perp}\right)
$$

and therefore $\mathcal{D}_{T,(P, Q)}(L) \neq 0$. To prove the converse, assume that $\mathcal{D}_{T,(P, Q)}(L)=0$. Then,

$$
\mathscr{S}_{T,(P, Q)}(L) \notin G L\left(N[T], R[T]^{\perp}\right)
$$

and hence,

$$
L_{11} \oplus \mathscr{S}_{T,(P, Q)}(L) \notin G L(U, V) .
$$

Consequently, by (3.5), $L \notin G L(U, V)$. This concludes the proof.

Thanks to this (local) notion of determinant, the generalized algebraic multiplicity can be also expressed in the vein of (2.4) even in an infinite-dimensional setting. Precisely, the next result holds.

Theorem 3.3. Assume $\mathfrak{L} \in \mathcal{A}_{\lambda_{0}}\left(\Omega_{\lambda_{0}}, \Phi_{0}(X, Y)\right)$, i.e. $\mathfrak{L} \in \mathcal{C}^{r}\left(\Omega, \Phi_{0}(U, V)\right.$ with $\lambda_{0} \in$ $\operatorname{Alg}_{\kappa}(\mathfrak{L})$ for some $1 \leq \kappa \leq r$ and $r \in \mathbb{N}$. Then,

$$
\chi\left[\mathfrak{L}, \lambda_{0}\right]=\operatorname{ord}_{\lambda_{0}} \mathcal{D}_{\mathfrak{L}\left(\lambda_{0}\right),(P, Q)}(\mathfrak{L}(\lambda))=\operatorname{ord}_{\lambda_{0}} \operatorname{det} \mathscr{S}_{\mathfrak{L}\left(\lambda_{0}\right),(P, Q)}(\mathfrak{L}(\lambda))
$$

for every pair $(P, Q)$ of $\mathfrak{L}\left(\lambda_{0}\right)$-projections.

Proof. First, we will show that, for every pair $(P, Q)$ of $\mathfrak{L}\left(\lambda_{0}\right)$-projections,

$$
\lambda_{0} \in \operatorname{Alg}_{\kappa}\left(\mathscr{S}_{\mathfrak{L}\left(\lambda_{0}\right),(P, Q)} \circ \mathfrak{L}\right) .
$$

Indeed, let $(P, Q)$ be a pair of $\mathfrak{L}\left(\lambda_{0}\right)$-projections. Then,

$$
\mathfrak{L}(\lambda)=\left(\begin{array}{ll}
L_{11}(\lambda) & L_{12}(\lambda) \\
L_{21}(\lambda) & L_{22}(\lambda)
\end{array}\right)
$$

where

$$
\begin{array}{ll}
L_{11}(\lambda):=Q \mathfrak{L}(\lambda)\left(I_{U}-P\right), & L_{12}(\lambda):=Q \mathfrak{L}(\lambda) P, \\
L_{21}(\lambda):=\left(I_{V}-Q\right) \mathfrak{L}(\lambda)\left(I_{U}-P\right), & L_{22}(\lambda):=\left(I_{V}-Q\right) \mathfrak{L}(\lambda) P .
\end{array}
$$


Since

$$
L_{11}\left(\lambda_{0}\right) \in G L\left(N\left[\mathfrak{L}\left(\lambda_{0}\right)\right]^{\perp}, R\left[\mathfrak{L}\left(\lambda_{0}\right)\right]\right),
$$

we can take $\Omega_{\lambda_{0}}$ sufficiently small so that

$$
L_{11}(\lambda) \in G L\left(N\left[\mathfrak{L}\left(\lambda_{0}\right)\right]^{\perp}, R\left[\mathfrak{L}\left(\lambda_{0}\right)\right]\right) \quad \text { for all } \lambda \in \Omega_{\lambda_{0}} .
$$

Thus, by (3.5), it becomes apparent that

$$
L_{11}(\lambda) \oplus \mathscr{S}_{\mathfrak{L}\left(\lambda_{0}\right),(P, Q)}(\mathfrak{L}(\lambda))=\mathfrak{L}_{1}^{-1}(\lambda) \mathfrak{L}(\lambda) \mathfrak{L}_{2}^{-1}(\lambda) \quad \text { for all } \quad \lambda \in \Omega_{\lambda_{0}}
$$

where

$$
\mathfrak{L}_{1}(\lambda):=\left(\begin{array}{cc}
I_{R\left[\mathfrak{L}\left(\lambda_{0}\right)\right]} & 0 \\
L_{21}(\lambda) L_{11}^{-1}(\lambda) & I_{R\left[\mathfrak{L}\left(\lambda_{0}\right)\right]^{\perp}}
\end{array}\right), \quad \mathfrak{L}_{2}(\lambda):=\left(\begin{array}{cc}
I_{N\left[\mathfrak{L}\left(\lambda_{0}\right)\right]^{\perp}} & L_{11}^{-1}(\lambda) L_{12}(\lambda) \\
0 & I_{N\left[\mathfrak{L}\left(\lambda_{0}\right)\right]}
\end{array}\right) .
$$

Therefore, inverting (3.8) yields

$$
L_{11}^{-1}(\lambda) \oplus\left[\mathscr{S}_{\mathfrak{L}\left(\lambda_{0}\right),(P, Q)}(\mathfrak{L}(\lambda))\right]^{-1}=\mathfrak{L}_{2}(\lambda) \mathfrak{L}^{-1}(\lambda) \mathfrak{L}_{1}(\lambda)
$$

for all $\lambda \in \Omega_{\lambda_{0}} \backslash\left\{\lambda_{0}\right\}$. Since

$$
\mathfrak{L}\left(\Omega_{\lambda_{0}} \backslash\left\{\lambda_{0}\right\}\right) \subset G L(U, V),
$$

by Theorem 3.2, we have that, for every $\lambda \in \Omega_{\lambda_{0}} \backslash\left\{\lambda_{0}\right\}$,

$$
\mathscr{S}_{\mathfrak{L}\left(\lambda_{0}\right),(P, Q)}(\mathfrak{L}(\lambda)) \in G L\left(N\left[\mathfrak{L}\left(\lambda_{0}\right)\right], R\left[\mathfrak{L}\left(\lambda_{0}\right)\right]^{\perp}\right) .
$$

On the one hand, since $\lambda_{0} \in \operatorname{Alg}_{\kappa}(\mathfrak{L})$, it is apparent that

$$
\left\|\mathfrak{L}_{2}(\lambda) \mathfrak{L}^{-1}(\lambda) \mathfrak{L}_{1}(\lambda)\right\| \leq C\left\|\mathfrak{L}^{-1}(\lambda)\right\| \leq \frac{C}{\left|\lambda-\lambda_{0}\right|^{\kappa}} .
$$

Moreover,

$$
\left\|\left[\mathscr{S}_{\mathfrak{L}\left(\lambda_{0}\right),(P, Q)}(\mathfrak{L}(\lambda))\right]^{-1}\right\| \leq\left\|L_{11}^{-1}(\lambda) \oplus\left[\mathscr{S}_{\mathfrak{L}\left(\lambda_{0}\right),(P, Q)}(\mathfrak{L}(\lambda))\right]^{-1}\right\| .
$$

Consequently, taking norms in (3.10), we may conclude from (3.12) and (3.11) that

$$
\left\|\left[\mathscr{S}_{\mathfrak{L}\left(\lambda_{0}\right),(P, Q)}(\mathfrak{L}(\lambda))\right]^{-1}\right\| \leq \frac{C}{\left|\lambda-\lambda_{0}\right|^{k}} \quad \text { for all } \lambda \in \Omega_{\lambda_{0}} \backslash\left\{\lambda_{0}\right\},
$$

which ends the proof of (3.7).

According to (3.8), we already know that

$$
\mathfrak{L}(\lambda)=\mathfrak{L}_{1}(\lambda)\left[L_{11}(\lambda) \oplus \mathscr{S}_{\mathfrak{L}\left(\lambda_{0}\right),(P, Q)}(\mathfrak{L}(\lambda))\right] \mathfrak{L}_{2}(\lambda),
$$

with $\mathfrak{L}_{1}(\lambda), \mathfrak{L}_{2}(\lambda) \in G L(U, V)$ for all $\lambda \in \Omega_{\lambda_{0}}$. Thus, by the properties of $\chi$ listed at the end of Section 3, it is apparent that

$$
\chi\left[\mathfrak{L}_{1}, \lambda_{0}\right]=\chi\left[\mathfrak{L}_{2}, \lambda_{0}\right]=0 .
$$

Moreover, since $L_{11}(\lambda) \in G L(U, V)$ for all $\lambda \in \Omega_{\lambda_{0}}$,

$$
\begin{aligned}
\chi\left[L_{11}(\lambda) \oplus\left(\mathscr{S}_{\mathfrak{L}\left(\lambda_{0}\right),(P, Q)} \circ \mathfrak{L}\right), \lambda_{0}\right] & =\chi\left[L_{11}(\lambda), \lambda_{0}\right]+\chi\left[\mathscr{S}_{\mathfrak{L}\left(\lambda_{0}\right),(P, Q)} \circ \mathfrak{L}, \lambda_{0}\right] \\
& =\chi\left[\mathscr{S}_{\mathfrak{L}\left(\lambda_{0}\right),(P, Q)} \circ \mathfrak{L}, \lambda_{0}\right] .
\end{aligned}
$$

Observe that $\chi\left[\mathscr{S}_{\mathfrak{L}\left(\lambda_{0}\right), \mathcal{P}} \circ \mathfrak{L}, \lambda_{0}\right]$ is well defined by (3.7). Therefore, applying the product formula to (3.13) yields

$$
\chi\left[\mathfrak{L}, \lambda_{0}\right]=\chi\left[\mathfrak{L}_{1}, \lambda_{0}\right]+\chi\left[L_{11} \oplus\left(\mathscr{S}_{\mathfrak{L}\left(\lambda_{0}\right),(P, Q)} \circ \mathfrak{L}\right), \lambda_{0}\right]+\chi\left[\mathfrak{L}_{2}, \lambda_{0}\right]=\chi\left[\mathscr{S}_{\mathfrak{L}\left(\lambda_{0}\right),(P, Q)} \circ \mathfrak{L}, \lambda_{0}\right] .
$$


Finally, since $\mathscr{S}_{\mathfrak{L}\left(\lambda_{0}\right),(P, Q)} \circ \mathfrak{L}$ is finite-dimensional and $L_{11}(\lambda)$ is an isomorphism, it follows from (2.4) and Definition 3.1 that

$$
\begin{aligned}
\chi\left[\mathscr{S}_{\mathfrak{L}\left(\lambda_{0}\right),(P, Q)} \circ \mathfrak{L}, \lambda_{0}\right] & =\operatorname{ord}_{\lambda_{0}} \mathcal{D} \mathscr{S}_{\mathfrak{L}\left(\lambda_{0}\right),(P, Q)}(\mathfrak{L}(\lambda)) \\
& =\operatorname{ord}_{\lambda_{0}} \mathcal{D} \mathscr{S}_{\mathfrak{L}\left(\lambda_{0}\right),(P, Q)}(\mathfrak{L}(\lambda)) \cdot \mathcal{D} L_{11}(\lambda) \\
& =\operatorname{ord}_{\lambda_{0}} \mathcal{D}_{\mathfrak{L}\left(\lambda_{0}\right),(P, Q)}(\mathfrak{L}(\lambda)) .
\end{aligned}
$$

Lastly, taking into account that

$$
\chi\left[\mathscr{S}_{\mathfrak{L}\left(\lambda_{0}\right),(P, Q)} \circ \mathfrak{L}, \lambda_{0}\right]=\operatorname{ord}_{\lambda_{0}} \operatorname{det} \mathscr{S}_{\mathfrak{L}\left(\lambda_{0}\right),(P, Q)}(\mathfrak{L}(\lambda)),
$$

the proof is completed.

To conclude this section, we are going to complete the proof of Theorem 1.1. Note that Theorem 3.3 establishes the validity of the first identity of (1.8). Assume $\mathfrak{L} \in$ $\mathcal{C}\left(\Omega, \Phi_{0}(U, V)\right)$, let $\lambda_{0} \in \Omega \cap \Sigma(\mathfrak{L})$ be an isolated eigenvalue, and consider a pair $(P, Q)$ of $\mathfrak{L}\left(\lambda_{0}\right)$-projections. According to (3.8), we already know that

$$
\mathfrak{L}(\lambda)=\mathfrak{L}_{1}(\lambda)\left(\begin{array}{cc}
L_{11}(\lambda) & 0 \\
0 & \mathscr{S}_{\mathfrak{L}\left(\lambda_{0}\right),(P, Q)}(\mathfrak{L}(\lambda))
\end{array}\right) \mathfrak{L}_{2}(\lambda)
$$

where

$$
\mathfrak{L}_{1}(\lambda):=\left(\begin{array}{cc}
I_{R\left[\mathfrak{L}\left(\lambda_{0}\right)\right]} & 0 \\
L_{21}(\lambda) L_{11}^{-1}(\lambda) & I_{R\left[\mathfrak{L}\left(\lambda_{0}\right)\right]^{\perp}}
\end{array}\right), \quad \mathfrak{L}_{2}(\lambda):=\left(\begin{array}{cc}
I_{N\left[\mathfrak{L}\left(\lambda_{0}\right)\right]^{\perp}} & L_{11}^{-1}(\lambda) L_{12}(\lambda) \\
0 & I_{N\left[\mathfrak{L}\left(\lambda_{0}\right)\right]}
\end{array}\right) .
$$

Thus, in a perforated neighborhood of $\lambda_{0}$ the inverse $\mathfrak{L}^{-1}(\lambda)$ is given through

$$
\left(\begin{array}{cc}
I_{N\left[\mathfrak{L}\left(\lambda_{0}\right)\right]^{\perp}} & -L_{11}^{-1}(\lambda) L_{12}(\lambda) \\
0 & I_{N\left[\mathfrak{L}\left(\lambda_{0}\right)\right]}
\end{array}\right)\left(\begin{array}{ccc}
L_{11}^{-1}(\lambda) & 0 \\
0 & \mathscr{S}_{\mathfrak{L}\left(\lambda_{0}\right),(P, Q)}^{-1}(\mathfrak{L}(\lambda))
\end{array}\right)\left(\begin{array}{cc}
I_{R\left[\mathfrak{L}\left(\lambda_{0}\right)\right]} & 0 \\
-L_{21}(\lambda) L_{11}^{-1}(\lambda) & I_{R\left[\mathfrak{L}\left(\lambda_{0}\right)\right]^{\perp}}
\end{array}\right) .
$$

Multiplying these operator matrices, it becomes apparent that

$$
\mathfrak{L}^{-1}=\left(\begin{array}{cc}
L_{11}^{-1}+L_{11}^{-1} L_{12} \mathscr{S}_{\mathfrak{L}\left(\lambda_{0}\right),(P, Q)}^{-1}(\mathfrak{L}) L_{21} L_{11}^{-1} & -L_{11}^{-1} L_{12} \mathscr{S}_{\mathfrak{L}\left(\lambda_{0}\right),(P, Q)}^{-1}(\mathfrak{L}) \\
-\mathscr{S}_{\mathfrak{L}\left(\lambda_{0}\right),(P, Q)}^{-1}(\mathfrak{L}) L_{21} L_{11}^{-1} & \mathscr{S}_{\mathfrak{L}\left(\lambda_{0}\right),(P, Q)}^{-1}(\mathfrak{L})
\end{array}\right) .
$$

Therefore, since

$$
\mathfrak{L}^{-1}(\lambda)=\left(\begin{array}{cc}
Q \mathfrak{L}^{-1}(\lambda)\left(I_{U}-P\right) & Q \mathfrak{L}^{-1}(\lambda) P \\
\left(I_{V}-Q\right) \mathfrak{L}^{-1}(\lambda)\left(I_{U}-P\right) & \left(I_{V}-Q\right) \mathfrak{L}^{-1}(\lambda) P
\end{array}\right),
$$

identifying the last entries of these two operator matrices, (1.7) holds. Therefore, by Theorem 3.3, (1.8) follows readily if $\mathfrak{L} \in \mathcal{A}_{\lambda_{0}}\left(\Omega_{\lambda_{0}}, \Phi_{0}(X, Y)\right)$. This ends the proof of Theorem 1.1.

\section{Proof of Theorem 1.2}

First, we will recover $\chi$ from the perspective of Nonlinear Analysis, by adopting the point of view of Bifurcation Theory. Actually, this was the methodology of Esquinas and LópezGómez [7] to construct $\chi$. In applications one is naturally lead to deal with nonlinear equations of the form

$$
\mathscr{F}(\lambda, u) \equiv \mathfrak{L}(\lambda) u+\mathfrak{N}(\lambda, u)=0,
$$

where $\mathfrak{L} \in \mathcal{C}^{1}\left(\Omega, \Phi_{0}(U, V)\right)$ for some subdomain $\Omega$ of $\mathbb{K}$, and $\mathfrak{N} \in \mathcal{C}^{1}(\Omega \times U, V)$ satisfies

$$
\mathfrak{N}(\lambda, 0)=0 \quad \text { and } \quad D_{u} \mathfrak{N}(\lambda, 0)=0
$$


for all $\lambda$ in a neighborhood of a given $\lambda_{0} \in \Omega$. Then, $\mathscr{F}(\lambda, 0)=0$ for $\lambda$ sufficiently close to $\lambda_{0}$ and, for any given pair $(P, Q)$ of $\mathfrak{L}\left(\lambda_{0}\right)$-projections, the equation (4.1) can be equivalently written as

$$
\left\{\begin{array}{l}
Q \mathfrak{L}(\lambda)(x+y)+Q \mathfrak{N}(\lambda, x+y)=0, \\
\left(I_{V}-Q\right) \mathfrak{L}(\lambda)(x+y)+\left(I_{V}-Q\right) \mathfrak{N}(\lambda, x+y)=0,
\end{array}\right.
$$

where we are setting

$$
x:=P u \in N\left[\mathfrak{L}\left(\lambda_{0}\right)\right], \quad y:=\left(I_{U}-P\right) u \in R\left[I_{U}-P\right] \equiv N\left[\mathfrak{L}\left(\lambda_{0}\right)\right]^{\perp} .
$$

Subsequently, we consider the map $\mathscr{G}$ defined by

$$
\mathscr{G}(\lambda, x, y):=Q \mathfrak{L}(\lambda)(x+y)+Q \mathfrak{N}(\lambda, x+y)
$$

for every $(\lambda, x, y)$ in a neighborhood, $\mathcal{O}$, of $\left(\lambda_{0}, 0,0\right)$ in

$$
\Omega \times N\left[\mathfrak{L}\left(\lambda_{0}\right)\right] \times N\left[\mathfrak{L}\left(\lambda_{0}\right)\right]^{\perp} \approx \Omega \times U .
$$

The map $\mathscr{G}$ is of class $\mathcal{C}^{1}(\mathcal{O})$ and it satisfies

$$
D_{y} \mathscr{G}(\lambda, 0,0)=Q \mathfrak{L}(\lambda)\left(I_{U}-P\right) \equiv L_{11}(\lambda)
$$

for $\lambda$ in a certain neighborhood, $\tilde{\Omega} \subset \Omega$, of $\lambda_{0}$, where we can assume that

$$
L_{11}(\lambda) \in G L\left(N\left[\mathfrak{L}\left(\lambda_{0}\right)\right]^{\perp}, R\left[\mathfrak{L}\left(\lambda_{0}\right)\right]\right) .
$$

Moreover, $\mathscr{G}(\lambda, 0,0)=0$ for all $\lambda \in \tilde{\Omega}$. Thus, since

$$
D_{y} \mathscr{G}(\lambda, 0,0)=L_{11}(\lambda) \in G L\left(N\left[\mathfrak{L}\left(\lambda_{0}\right)\right]^{\perp}, R\left[\mathfrak{L}\left(\lambda_{0}\right)\right]\right),
$$

by the Implicit Function Theorem, there exist a neighborhood, $\mathcal{U}$, of $\left(\lambda_{0}, 0\right)$ in $\mathbb{K} \times N\left[\mathfrak{L}\left(\lambda_{0}\right)\right]$ and a map $\mathscr{Y} \in \mathcal{C}^{1}\left(\mathcal{U}, R\left[\mathfrak{L}\left(\lambda_{0}\right)\right]\right)$ such that

$$
\mathscr{Y}(\lambda, 0)=0 \quad \text { and } \quad \mathscr{G}(\lambda, x, \mathscr{Y}(\lambda, x))=0
$$

for all $(\lambda, x) \in \mathcal{U}$, and these are the unique zeroes of $\mathscr{G}$ in $\mathcal{U}$. So, $\mathscr{Y}(\lambda, 0)=0$ for $\lambda$ sufficiently close to $\lambda_{0}$, because $\mathscr{G}(\lambda, 0,0)=0$. Furthermore, by the definition of $\mathscr{G}$, we have that, for every $(\lambda, x) \in \mathcal{U}$,

$$
Q \mathfrak{L}(\lambda)(x+\mathscr{Y}(\lambda, x))+Q \mathfrak{N}(\lambda, x+\mathscr{Y}(\lambda, x))=0 .
$$

Thus, thanks to (4.2), differentiating (4.4) with respect to $x$ yields to

$$
Q \mathfrak{L}(\lambda)\left(x+D_{x} \mathscr{Y}(\lambda, 0) x\right)=0 \quad \text { for all } x \in N\left[\mathfrak{L}\left(\lambda_{0}\right)\right] .
$$

Equivalently,

$$
Q \mathfrak{L}(\lambda)\left(I_{U}-P\right) D_{x} \mathscr{Y}(\lambda, 0) x=-Q \mathfrak{L}(\lambda) P x \quad \text { for all } x \in N\left[\mathfrak{L}\left(\lambda_{0}\right)\right] .
$$

Consequently, for sufficiently small $\tilde{\Omega}$, we find that, for every $\lambda \in \tilde{\Omega}$,

$$
D_{x} \mathscr{Y}(\lambda, 0)=-\left[Q \mathfrak{L}(\lambda)\left(I_{U}-P\right)\right]^{-1} Q \mathfrak{L}(\lambda) P=-L_{11}^{-1}(\lambda) L_{12}(\lambda) .
$$

Consequently, substituting $y$ by $\mathscr{Y}(\lambda, x)$ into the second equation of (4.3), it turns out that solving (4.1) locally at $(\lambda, u)=\left(\lambda_{0}, 0\right)$ is equivalent to solve the equation

$$
\left(I_{V}-Q\right) \mathfrak{L}(\lambda)(x+\mathscr{Y}(\lambda, x))+\left(I_{V}-Q\right) \mathfrak{N}(\lambda, x+\mathscr{Y}(\lambda, x))=0
$$

in a neighborhood of $\left(\lambda_{0}, 0\right)$ in $\mathbb{K} \times N\left[\mathfrak{L}\left(\lambda_{0}\right)\right]$. Equation (4.6) is often referred to as the bifurcation equation of (4.1) at $\left(\lambda_{0}, 0\right)$. According to [20, Le. 3.1.1], $(\lambda, u)=\left(\lambda_{0}, 0\right)$ is a bifurcation point of (4.1) from $(\lambda, u)=(\lambda, 0)$ if and only if $(\lambda, x)=\left(\lambda_{0}, 0\right)$ is a bifurcation point of (4.5) from $(\lambda, x)=(\lambda, 0)$. As the linearization of the left hand side of (4.6) at $(\lambda, x)=(\lambda, 0)$ is given by the linear operator

$$
\mathscr{B}(\lambda) x:=\left(I_{V}-Q\right) \mathfrak{L}(\lambda)\left(x+D_{x} \mathscr{Y}(\lambda, 0) x\right) \quad \text { for all } x \in N[\mathfrak{L}(\lambda)],
$$


and, due to (4.5), $\mathscr{B}(\lambda)$ can be expressed as

$$
\mathscr{B}(\lambda):=\left(I_{V}-Q\right) \mathfrak{L}(\lambda)\left[P-\left(I_{U}-P\right) L_{11}^{-1}(\lambda) L_{12}(\lambda)\right],
$$

by the definitions of the $L_{i j}(\lambda)$ 's, it becomes apparent that

$$
\mathscr{B}(\lambda):=L_{22}(\lambda)-L_{21}(\lambda) L_{11}^{-1}(\lambda) L_{12}(\lambda)=\mathscr{S}_{\mathfrak{L}(\lambda),(P, Q)}(\mathfrak{L}(\lambda)) .
$$

Therefore, the first part of Theorem 1.2 and, in particular, (1.9) is proven. Naturally, (1.10) follows readily by combining (1.9) with Theorem 1.1. The equivalence between (b), (c), (d), and (e) is a direct consequence from (1.8) and (1.10), and the equivalence of (a) and (b) is [20, Th. 4.3.4].

Finally, suppose that $\chi\left[\mathfrak{L}, \lambda_{0}\right]$ is an odd integer. Then, due to (1.10), det $\mathscr{B}(\lambda)$ changes sign as $\lambda$ crosses $\lambda_{0}$. Hence, also the Brouwer degree of $(\lambda, 0)$ changes as $\lambda$ crosses $\lambda_{0}$. Thus, thanks to [20, Th. 6.2.1], the bifurcation equation (4.6) possesses a (local) continuum of non-trivial solutions emanating from $(\lambda, x)=(\lambda, 0)$ at $\lambda_{0}$. Naturally, by the local structure of the solutions of (4.1) in a neighborhood of $(\lambda, u)=\left(\lambda_{0}, 0\right)$, it becomes apparent that (4.1) also has a (local) continuum of non-trivial solutions emanating from $(\lambda, u)=(\lambda, 0)$ at $\lambda=\lambda_{0}$. Consequently, Zorn's lemma shows the existence of the component $\mathscr{C}$. This ends the proof of Theorem 1.2 .

\section{The Classical multiplicity from a GeOMEtrical PerspeCtive}

The main goal of this section is establishing a, rather hidden, bi-association between the classical algebraic multiplicity and the concept of intersection index of varieties in Algebraic Geometry. Naturally, this might open new challenging perspectives in both fields, besides incrementing their number of potential applications. The next, and final, section will extend the findings of this one to cover the generalized algebraic multiplicity $\chi$. As for algebraic purposes one needs to work in algebraically closed fields, throughout the rest of this paper we will assume that $\mathbb{K}=\mathbb{C}$.

Subsequently, we identify $\mathcal{L}\left(\mathbb{C}^{N}\right) \simeq \mathbb{C}^{N^{2}}$ and regard the determinant map,

$$
\mathcal{D}: \mathcal{L}\left(\mathbb{C}^{N}\right) \rightarrow \mathbb{C}, \quad \mathcal{D}(T):=\operatorname{det} T \text { for every } T \in \mathcal{L}\left(\mathbb{C}^{N}\right),
$$

as an homogeneous polynomial map of degree $N$. Using this identification, every $T \in$ $\mathcal{L}\left(\mathbb{C}^{N}\right)$ can be expressed as en element of $\mathbb{C}^{N^{2}}$ through the identification

$$
T=\left(\begin{array}{cccc}
x_{1} & x_{2} & \cdots & x_{N} \\
x_{N+1} & x_{N+2} & \cdots & x_{2 N} \\
\vdots & \vdots & \ddots & \vdots \\
x_{N^{2}-N+1} & x_{N^{2}-N+2} & \cdots & x_{N^{2}}
\end{array}\right)=\left(x_{1}, x_{2}, \ldots, x_{N^{2}-1}, x_{N^{2}}\right) .
$$

Then, for every $1 \leq k \leq N^{2}$, we define the $k$-th differential functional of the determinant map at a given operator $L \in \mathcal{L}\left(\mathbb{C}^{N}\right)$ as the map $\mathcal{D}^{k}(L): \mathcal{L}\left(\mathbb{C}^{N}\right) \rightarrow \mathbb{C}$ defined by

$$
\mathcal{D}^{k}(L)[T]:=\sum_{1 \leq j_{1}, j_{2}, \cdots, j_{k} \leq N^{2}}\left(\frac{\partial^{k} \mathcal{D}}{\partial x_{j_{1}} \partial x_{j_{2}} \cdots \partial x_{j_{k}}}\right)(L) \cdot x_{j_{1}} x_{j_{2}} \cdots x_{j_{k}}
$$

for all $T \in \mathcal{L}\left(\mathbb{C}^{N}\right)$. Note that, for any given $L \in \mathcal{L}\left(\mathbb{C}^{N}\right)$, the Taylor expansion of the determinant map, $\mathcal{D}$, centered at $L$, is given through

$$
\mathcal{D}(T)=\mathcal{D}(L)+\sum_{k=1}^{N^{2}} \frac{1}{k !} \mathcal{D}^{k}(L)[T-L] \quad \text { for all } T \in \mathcal{L}\left(\mathbb{C}^{N}\right) .
$$


In particular, $\mathcal{D}^{k}(L)$ can be viewed as an homogeneous polynomial of degree $k$. The associated algebraic variety

$$
V\left(\mathcal{D}^{k}(L)\right)=\left(\mathcal{D}^{k}(L)\right)^{-1}(0)
$$

will be subsequently refereed to as the $k$-th tangent variety of the determinant map at $L \in \mathcal{L}\left(\mathbb{C}^{N}\right)$, and will be denoted by $\mathscr{T}_{L}^{k} \mathcal{D}$. As $\mathscr{T}_{L}^{1} \mathcal{D}$ is the tangent space to $D^{-1}(0)$ at $L$, the variety $\mathscr{T}_{L}^{k} \mathcal{D}$ can be viewed as a generalization of the classical tangent space at a given point. The following technical result can be easily obtained from the chain rule.

Lemma 5.1. Let $T \in \mathcal{L}\left(\mathbb{C}^{N}\right)$ and $\mathfrak{L}(\lambda)=\lambda I_{N}-T$ for all $\lambda \in \mathbb{C}$. Then, for every $r \in \mathbb{N}$, the following identity holds

$$
\frac{d^{r}}{d \lambda^{r}}(\mathcal{D} \mathfrak{L}(\lambda)) \equiv(\mathcal{D} \mathfrak{L})^{(r)}(\lambda)=\mathcal{D}^{r}(\mathfrak{L}(\lambda))\left[I_{N}\right]
$$

Proof. For notational convenience, we will identify $x_{i, j}$ with $x_{(i-1) N+j}$ for any $N \times N$ matrix $\left(x_{i, j}\right)_{1 \leq i, j \leq N}$. By definition,

$$
\mathcal{D}^{r}(\mathfrak{L}(\lambda))\left[I_{N}\right]=\sum_{1 \leq i_{1}, i_{2}, \cdots, i_{r} \leq N}\left(\frac{\partial^{r} \mathcal{D}}{\partial x_{i_{1}, i_{1}} \partial x_{i_{2}, i_{2}} \cdots \partial x_{i_{r}, i_{r}}}\right)(\mathfrak{L}(\lambda)) .
$$

Thus, (5.1) can be equivalently written in the form

$$
(\mathcal{D} \mathfrak{L})^{(r)}(\lambda)=\sum_{1 \leq i_{1}, i_{2}, \cdots, i_{r} \leq N}\left(\frac{\partial^{r} \mathcal{D}}{\partial x_{i_{1}, i_{1}} \partial x_{i_{2}, i_{2}} \cdots \partial x_{i_{r}, i_{r}}}\right)(\mathfrak{L}(\lambda)) .
$$

The proof of (5.2) will proceed by induction on $r$. Suppose $r=1$. Then, since $\mathfrak{L}^{\prime}(\lambda)=I_{N}$, we have that

$$
(\mathcal{D} \mathfrak{L})^{\prime}(\lambda)=\nabla \mathcal{D}(\mathfrak{L}(\lambda)) \circ \mathfrak{L}^{\prime}(\lambda)=\sum_{i=1}^{N^{2}} \frac{\partial \mathcal{D}}{\partial x_{i}}(\mathfrak{L}(\lambda)) \cdot\left[\mathfrak{L}^{\prime}(\lambda)\right]_{i}=\sum_{i_{1}=1}^{N} \frac{\partial \mathcal{D}}{\partial x_{i_{1}, i_{1}}}(\mathfrak{L}(\lambda)),
$$

where $\left[\mathfrak{L}^{\prime}(\lambda)\right]_{i}$ is the $i$-th term of the matrix $\mathfrak{L}^{\prime}(\lambda)$. Thus, (5.2) holds for $r=1$. Now, suppose that (5.2) is satisfied for some $r=n-1$ with $n \geq 2$, i.e.,

$$
(\mathcal{D} \mathfrak{L})^{(n-1)}(\lambda)=\sum_{1 \leq i_{1}, i_{2}, \cdots, i_{n-1} \leq N}\left(\frac{\partial^{n-1} \mathcal{D}}{\partial x_{i_{1}, i_{1}} \partial x_{i_{2}, i_{2}} \cdots \partial x_{i_{n-1}, i_{n-1}}}\right)(\mathfrak{L}(\lambda)) .
$$

Then, differentiating with respect to $\lambda$ yields

$$
\begin{aligned}
(\mathcal{D} \mathfrak{L})^{(n)}(\lambda) & =\frac{d}{d \lambda} \sum_{1 \leq i_{1}, i_{2}, \cdots, i_{n-1} \leq N}\left(\frac{\partial^{n-1} \mathcal{D}}{\partial x_{i_{1}, i_{1}} \partial x_{i_{2}, i_{2}} \cdots \partial x_{i_{n-1}, i_{n-1}}}\right)(\mathfrak{L}(\lambda)) \\
& =\sum_{1 \leq i_{1}, i_{2}, \cdots, i_{n-1} \leq N} \frac{d}{d \lambda}\left[\left(\frac{\partial^{n-1} \mathcal{D}}{\partial x_{i_{1}, i_{1}} \partial x_{i_{2}, i_{2}} \cdots \partial x_{i_{n-1}, i_{n-1}}}\right)(\mathfrak{L}(\lambda))\right] \\
& =\sum_{1 \leq i_{1}, i_{2}, \cdots, i_{n-1} \leq N} \sum_{j=1}^{N^{2}}\left(\frac{\partial^{n} \mathcal{D}}{\partial x_{i_{1}, i_{1}} \partial x_{i_{2}, i_{2}} \cdots \partial x_{i_{n-1}, i_{n-1}} \partial x_{j}}\right)(\mathfrak{L}(\lambda)) \cdot\left[\mathfrak{L}^{\prime}(\lambda)\right]_{j} \\
& =\sum_{1 \leq i_{1}, i_{2}, \cdots, i_{n} \leq N}\left(\frac{\partial^{n} \mathcal{D}}{\partial x_{i_{1}, i_{1}} \partial x_{i_{2}, i_{2}} \cdots \partial x_{i_{n}, i_{n}}}\right)(\mathfrak{L}(\lambda)) .
\end{aligned}
$$

This concludes the proof. 
The following result, illustrated by Figure1, gives a geometrical meaning to the classical algebraic multiplicity of an eigenvalue of a linear operator. As illustrated by Figure1, the algebraic multiplicity provides us with the minimal integer $\mathfrak{m}$ for which the straight line $\lambda I_{N}$ is not contained within the $\mathfrak{m}$-th tangent variety of the determinant map at $\mathfrak{L}\left(\lambda_{0}\right)$, which has been denoted by $\mathscr{T}_{\mathfrak{L}\left(\lambda_{0}\right)}^{\mathfrak{m}} \mathcal{D}$. Actually, Figure 1 illustrates an admissible case when $\lambda I_{N}$ lies entirely within the tangent variety $\mathscr{T}_{\mathfrak{L}\left(\lambda_{0}\right)}^{1} \mathcal{D}$ and hence,

$$
\mathfrak{m}=\chi\left[\lambda I_{N}-T, \lambda_{0}\right] \geq 2 .
$$

Theorem 5.2. Let $T \in \mathcal{L}\left(\mathbb{C}^{N}\right)$, pick $\lambda_{0} \in \sigma(T)$, and consider the straight line

$$
\mathfrak{L}(\lambda)=\lambda I_{N}-T, \quad \lambda \in \mathbb{C} .
$$

Then, $\mathfrak{m}:=\chi\left[\mathfrak{L}, \lambda_{0}\right]$ is the minimal integer $\mathfrak{m} \geq 1$ for which the line $\lambda I_{N}$ is contained in $\mathscr{T}_{\mathfrak{L}\left(\lambda_{0}\right)}^{i} \mathcal{D}$ for every $1 \leq i<\mathfrak{m}$, but not for $i=\mathfrak{m}$.

Proof. By (5.3) $), \mathfrak{m}=\chi\left[\mathfrak{L}, \lambda_{0}\right]$ if and only if

$$
(\mathcal{D} \mathfrak{L})^{(i)}\left(\lambda_{0}\right)=0 \quad \text { for each } \quad i \in\{1, \ldots, \mathfrak{m}-1\} \text { and }(\mathcal{D} \mathfrak{L})^{(\mathfrak{m})}\left(\lambda_{0}\right) \neq 0 .
$$

Therefore, owing to (5.1), it becomes apparent that

$$
\mathcal{D}^{i}\left(\mathfrak{L}\left(\lambda_{0}\right)\right)\left[I_{N}\right]=0 \quad \text { for each } \quad i \in\{1, \ldots, \mathfrak{m}-1\} \text { and } \mathcal{D}^{\mathfrak{m}}\left(\mathfrak{L}\left(\lambda_{0}\right)\right)\left[I_{N}\right] \neq 0 .
$$

We conclude the proof by noticing that the line $\lambda I_{N}$ is contained in the variety

$$
\mathscr{T}_{\mathfrak{L}\left(\lambda_{0}\right)}^{i} \mathcal{D}=\left(\mathcal{D}^{i}\left(\mathfrak{L}\left(\lambda_{0}\right)\right)\right)^{-1}(0)
$$

if and only if

$$
\mathcal{D}^{i}\left(\mathfrak{L}\left(\lambda_{0}\right)\right)\left[\lambda I_{N}\right]=0 \text { for all } \lambda \in \mathbb{C} .
$$

As the map $\mathcal{D}^{i}\left(\mathfrak{L}\left(\lambda_{0}\right)\right)$ is linear, this occurs if and only if

$$
\mathcal{D}^{i}\left(\mathfrak{L}\left(\lambda_{0}\right)\right)\left[I_{N}\right]=0 .
$$

The proof is complete.

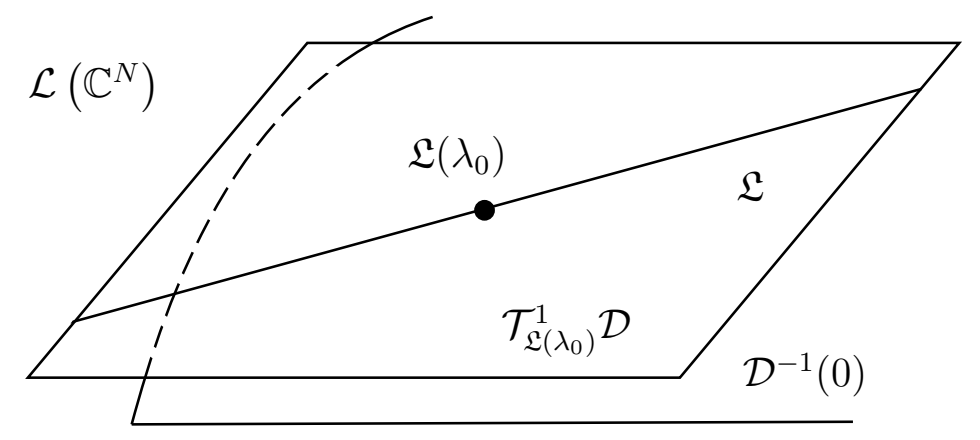

Figure 1. Geometrical Meaning of Classical Algebraic Multiplicity

Theorem 5.2 leads us to think of the existence of a sharp connection between Spectral Theory and Algebraic Geometry, which had been already stated in Theorem 1.3. The rest of this section is devoted to deliver a proof of this theorem.

Let $T \in \mathcal{L}\left(\mathbb{C}^{N}\right)$, pick $\lambda_{0} \in \sigma(T)$, and consider the straight line

$$
\mathfrak{L}(\lambda)=\lambda I_{N}-T, \quad \lambda \in \mathbb{C} .
$$


As usual in this section, we express the operator $T \in \mathcal{L}\left(\mathbb{C}^{N}\right)$ in matrix form

$$
T \sim\left(\begin{array}{cccc}
t_{1} & t_{2} & \cdots & t_{N} \\
t_{N+1} & t_{N+2} & \cdots & t_{2 N} \\
\vdots & \vdots & \ddots & \vdots \\
t_{N^{2}-N+1} & t_{N^{2}-N+2} & \cdots & t_{N^{2}}
\end{array}\right),
$$

i.e., setting $T=\left(t_{i, j}\right)_{1 \leq i, j \leq N}$, we are identifying $t_{i, j}$ with $t_{(i-1) N+j}$.

Naturally, the parametric equations of the line $\mathfrak{L}(\mathbb{C})$ in $\mathbb{C}^{N^{2}}$ are the following ones:

$$
\begin{cases}x_{i}=\lambda-t_{i} & \text { for } i \in\{(m-1) N+m: m \in\{1,2, \ldots, N\}\}, \\ x_{i}=-t_{i} & \text { for } i \in\left\{1,2, \ldots, N^{2}\right\} \backslash\{(m-1) N+m: m \in\{1,2, \ldots, N\}\} .\end{cases}
$$

Thus, by a simple computation involving the following identity

$$
\lambda=x_{1}+t_{1},
$$

it is easily seen that $\mathfrak{L}(\mathbb{C})$ is the algebraic variety generated by the polynomials

$$
\begin{cases}\mathfrak{P}_{i-1} \equiv x_{i}-x_{1}+t_{i}-t_{1} & \text { for } i \in\{(m-1) N+m: m \in\{2, \ldots, N\}\} \\ \mathfrak{P}_{i-1} \equiv x_{i}+t_{i} & \text { for } i \in\left\{1,2, \ldots, N^{2}\right\} \backslash\{(m-1) N+m: m \in\{1,2, \ldots, N\}\}\end{cases}
$$

In other words,

$$
\mathfrak{L}(\mathbb{C})=V\left(\mathfrak{P}_{1}, \ldots, \mathfrak{P}_{N^{2}-1}\right),
$$

i.e., the implicit equations of $\mathfrak{L}(\mathbb{C})$ are

$$
\begin{cases}x_{i}-x_{1}+t_{i}-t_{1}=0 & \text { for } i \in\{(m-1) N+m: m \in\{2, \ldots, N\}\}, \\ x_{i}+t_{i}=0 & \text { for } i \in\left\{1,2, \ldots, N^{2}\right\} \backslash\{(m-1) N+m: m \in\{1,2, \ldots, N\}\} .\end{cases}
$$

According to Theorem 18.18 of Eisenbud [4], the determinantal variety $\mathcal{D}^{-1}(0)$ is CohenMacaulay, as well as $\mathfrak{L}(\mathbb{C})$, because it is smooth (see [4, Sect. 18.2]). Therefore, by Eisenbud and Harris [5, p. 48],

$$
i\left(\mathcal{D}^{-1}(0), \mathfrak{L}(\mathbb{C}) ; \mathfrak{L}\left(\lambda_{0}\right)\right)=\ell_{\mathcal{O}}(\mathcal{O} /(\mathfrak{D}, \mathfrak{P}))
$$

(see (1.12)) , where $\mathcal{O} \equiv \mathcal{O}_{\mathfrak{L}\left(\lambda_{0}\right), \mathbb{C}^{N^{2}}}$ stands for the local ring of $\mathbb{C}^{N^{2}}$ at $\mathfrak{L}\left(\lambda_{0}\right), \mathfrak{D}$ is the ideal generated by the determinant map,

$$
\mathfrak{D}:=\left(\mathcal{D}\left(x_{1}, x_{2}, \cdots, x_{N^{2}}\right)\right)
$$

$\mathfrak{P}$ stands for the ideal generated by the polynomials $\mathfrak{P}_{j}, 1 \leq j \leq N^{2}-1$, and $\ell_{\mathcal{O}}$ is the length of the module $\mathcal{O} /(\mathfrak{D}, \mathfrak{P})$ over the ring $\mathcal{O}$. By (5.4),

$$
\begin{aligned}
(\mathfrak{D}, \mathfrak{P}) & =\left(\mathcal{D}\left(x_{1}, x_{2}, \cdots, x_{N^{2}}\right), \mathfrak{P}_{1}, \mathfrak{P}_{2}, \cdots, \mathfrak{P}_{N^{2}-1}\right) \\
& =\left(\mathcal{D}\left[\left(x_{1}+t_{1}\right) I_{N}-T\right], \mathfrak{P}_{1}, \mathfrak{P}_{2}, \cdots, \mathfrak{P}_{N^{2}-1}\right) \\
& =\left(\mathcal{D} \mathfrak{L}\left(x_{1}+t_{1}\right), \mathfrak{P}_{1}, \mathfrak{P}_{2}, \cdots, \mathfrak{P}_{N^{2}-1}\right) .
\end{aligned}
$$

On the other hand, by the definition of the classical algebraic geometry, $\mathfrak{m}=\chi\left[\lambda I_{N}-T, \lambda_{0}\right]$, the following identity holds

$$
\mathcal{D} \mathfrak{L}\left(x_{1}+t_{1}\right)=\left(x_{1}+t_{1}-\lambda_{0}\right)^{\mathfrak{m}} f\left(x_{1}+t_{1}\right)
$$

for some polynomial $f$ such that $f\left(\lambda_{0}\right) \neq 0$. Thus, since $f\left(x_{1}+t_{1}\right)$ is a unit in the local $\operatorname{ring} \mathcal{O}_{\mathfrak{L}\left(\lambda_{0}\right), \mathbb{C}^{N^{2}}}$, we find that

$$
(\mathfrak{D}, \mathfrak{P})=\left(\left(x_{1}+t_{1}-\lambda_{0}\right)^{\mathfrak{m}}, \mathfrak{P}_{1}, \mathfrak{P}_{2}, \cdots, \mathfrak{P}_{N^{2}-1}\right) .
$$

On the other hand, by a routine calculation,

$$
V\left(\left(x_{1}+t_{1}-\lambda_{0}\right)^{\mathfrak{m}}, \mathfrak{P}_{1}, \mathfrak{P}_{2}, \cdots, \mathfrak{P}_{N^{2}-1}\right)=\left\{\mathfrak{L}\left(\lambda_{0}\right)\right\} .
$$


Therefore, by, e.g., Proposition 6 of Fulton [9], we get the isomorphism

$$
\frac{\mathcal{O}_{\mathfrak{L}\left(\lambda_{0}\right), \mathbb{C}^{N^{2}}}}{(\mathfrak{D}, \mathfrak{P})} \simeq \frac{\mathbb{C}\left[x_{1}, x_{2}, \ldots, x_{N^{2}}\right]}{(\mathfrak{D}, \mathfrak{P})}
$$

Moreover, since the residue field of $\mathcal{O}$ is $\mathbb{C}$ and $\mathfrak{m} \cdot \mathcal{O} /(\mathfrak{D}, \mathfrak{P})=0$, where $\mathfrak{m}$ is the maximal ideal of $\mathcal{O}$, necessarily, by Proposition 5.9 of Bump [2],

$$
\ell_{\mathcal{O}}(\mathcal{O} /(\mathfrak{D}, \mathfrak{P}))=\operatorname{dim}_{\mathbb{C}}(\mathcal{O} /(\mathfrak{D}, \mathfrak{P})) \text {. }
$$

Since in $\mathbb{C}\left[x_{1}, x_{2}, \ldots, x_{N^{2}}\right] /(\mathfrak{D}, \mathfrak{P})$ the following $N^{2}$ identities hold true

$$
\begin{cases}\left(x_{1}+t_{1}-\lambda_{0}\right)^{\mathfrak{m}}=0 & \text { for } i \in\{(m-1) n+m: m \in\{2, \ldots, n\}\}, \\ x_{i}=x_{1}+t_{1}-t_{i} & \text { for } i \in\left\{1,2, \ldots, n^{2}\right\} \backslash\{(m-1) n+m: m \in\{1,2, \ldots, n\}\}, \\ x_{i}=-t_{i} & \end{cases}
$$

it is apparent, from the Newton binomial, that $x_{1}^{\mathfrak{m}}$ depends linearly on $x_{1}^{j}, 0 \leq j \leq \mathfrak{m}-1$, as well as the $x_{i}$ 's for all $i \in\left\{2, \ldots, N^{2}\right\}$. Therefore,

$$
\frac{\mathbb{C}\left[x_{1}, x_{2}, \ldots, x_{N^{2}}\right]}{(\mathfrak{D}, \mathfrak{P})}=\left\langle 1, x_{1}, x_{1}^{2}, \cdots, x_{1}^{\mathfrak{m}-1}\right\rangle_{\mathbb{C}}
$$

and consequently,

$$
\begin{aligned}
i\left(\mathcal{D}^{-1}(0), \mathfrak{L}(\mathbb{R}) ; \mathfrak{L}\left(\lambda_{0}\right)\right) & =\ell_{\mathcal{O}}(\mathcal{O} /(\mathfrak{D}, \mathfrak{P}))=\operatorname{dim}_{\mathbb{C}}(\mathcal{O} /(\mathfrak{D}, \mathfrak{P})) \\
& =\operatorname{dim}_{\mathbb{C}}\left(\mathbb{C}\left[x_{1}, x_{2}, \ldots, x_{N^{2}}\right] /(\mathfrak{D}, \mathfrak{P})\right)=\mathfrak{m} .
\end{aligned}
$$

This ends the proof of Theorem 1.3 .

\section{Proof of Theorem 1.4}

Thanks to the existence of the local Smith form for general analytic curves, $\mathfrak{L}$, at isolated singular values, $\lambda_{0}$, the algebraic multiplicity of these curves, $\chi\left[\mathfrak{L}, \lambda_{0}\right]$, can be also expressed as the intersection index of two algebraic varieties, as already stated by Theorem 1.4. This section consists of the proof of that theorem.

Let $\mathfrak{L} \in \mathcal{A}_{\lambda_{0}}\left(\Omega_{\lambda_{0}}, \Phi_{0}(X, Y)\right)$ be, and fix a pair $(P, Q)$ of $\mathfrak{L}\left(\lambda_{0}\right)$-projections. Then, thanks to Theorem 3.3. we have that

$$
\begin{aligned}
\chi\left[\mathfrak{L}, \lambda_{0}\right] & =\operatorname{ord}_{\lambda_{0}} \mathcal{D}_{\mathfrak{L}\left(\lambda_{0}\right),(P, Q)}(\mathfrak{L}(\lambda))=\operatorname{ord}_{\lambda_{0}} \mathcal{D} \mathscr{S}_{\mathfrak{L}\left(\lambda_{0}\right),(P, Q)}(\mathfrak{L}(\lambda)) \\
& =\operatorname{ord}_{\lambda_{0}} \mathcal{D}\left[L_{22}(\lambda)-L_{21}(\lambda) L_{11}^{-1}(\lambda) L_{12}(\lambda)\right] .
\end{aligned}
$$

For the sake of notation, let us identify

$$
\mathcal{L}\left(N\left[\mathfrak{L}\left(\lambda_{0}\right)\right], R\left[\mathfrak{L}\left(\lambda_{0}\right)\right]^{\perp}\right) \simeq \mathcal{L}\left(\mathbb{C}^{N}\right) \quad \text { with } \quad N:=\operatorname{dim} N\left[\mathfrak{L}\left(\lambda_{0}\right)\right] .
$$

According to (3.7), we already know that

$$
\lambda_{0} \in \operatorname{Alg}_{\kappa}\left(\mathscr{S}_{\mathfrak{L}\left(\lambda_{0}\right),(P, Q)} \circ \mathfrak{L}\right)
$$

for some $1 \leq \kappa \leq r$, where $\mathcal{C}^{r}$ is the class of regularity of $\mathfrak{L}(\lambda)$. Thus, by Theorem 7.8.1 of López-Gómez and Mora-Corral [23], $\mathscr{S}_{\mathfrak{L}\left(\lambda_{0}\right), \mathcal{P}} \circ \mathfrak{L}$ admits a local Smith form at $\lambda_{0}$, i.e., there exist a neighborhood, $\Omega_{\lambda_{0}}$, of $\lambda_{0}$ such that $\Omega_{\lambda_{0}} \subset \Omega$, two invertible curves $\mathfrak{E}, \mathfrak{F} \in \mathcal{C}\left(\Omega_{\lambda_{0}}, G L\left(\mathbb{C}^{N}\right)\right)$, and $N$ positive integers

$$
\kappa_{1} \geq \cdots \geq \kappa_{N} \geq 1
$$

such that

$$
\mathscr{S}_{\mathfrak{L}\left(\lambda_{0}\right),(P, Q)}(\mathfrak{L}(\lambda))=\mathfrak{E}(\lambda) \cdot \mathfrak{P}(\lambda) \cdot \mathfrak{F}(\lambda) \text { for all } \lambda \in \Omega_{\lambda_{0}},
$$

where $\mathfrak{P}(\lambda)$ is the matrix of order $n$ defined by

$$
\mathfrak{P}(\lambda):=\operatorname{diag}\left(\left(\lambda-\lambda_{0}\right)^{\kappa_{1}}, \ldots,\left(\lambda-\lambda_{0}\right)^{\kappa_{N}}\right),
$$


which is the local Smith form of the Schur operator of $\mathfrak{L}(\lambda)$ at $\lambda_{0}$.

On the other hand, according to Lemma 10.1.1 of López-Gómez and Mora-Corral [23], there exist an integer $M \geq N$, two invertible matrix polynomial curves $\mathfrak{P}_{1}, \mathfrak{P}_{2} \in$ $\mathcal{H}\left(\Omega_{\lambda_{0}}, G L\left(\mathbb{C}^{M}\right)\right)$, and an operator $\mathscr{L} \in \mathcal{L}\left(\mathbb{C}^{M}\right)$ such that

$$
\mathfrak{P}(\lambda) \oplus I_{M-N}=\mathfrak{P}_{1}(\lambda) \cdot\left(\lambda I_{M}-\mathscr{L}\right) \cdot \mathfrak{P}_{2}(\lambda) \text { for all } \lambda \in \Omega_{\lambda_{0}} .
$$

Hence, bringing together (6.1) and (6.3), it becomes apparent that there are two finite dimensional curves $\mathfrak{E}_{1}, \mathfrak{F}_{1} \in \mathcal{C}\left(\Omega_{\lambda_{0}}, G L\left(\mathbb{C}^{M}\right)\right)$ such that

$$
\mathscr{S}_{\mathfrak{L}\left(\lambda_{0}\right),(P, Q)}(\mathfrak{L}(\lambda)) \oplus I_{M-N}=\mathfrak{C}_{1}(\lambda) \cdot\left(\lambda I_{M}-\mathscr{L}\right) \cdot \mathfrak{F}_{1}(\lambda) \text { for all } \lambda \in \Omega_{\lambda_{0}} .
$$

Consequently, taking determinants on both sides of (6.4) it is apparent that

$$
\begin{aligned}
\mathcal{D}\left[\mathscr{S}_{\mathfrak{L}\left(\lambda_{0}\right),(P, Q)}(\mathfrak{L}(\lambda))\right] & =\mathcal{D}\left(\mathfrak{E}_{1}(\lambda) \cdot\left(\lambda I_{M}-\mathscr{L}\right) \cdot \mathfrak{F}_{1}(\lambda)\right) \\
& =\mathcal{D}\left(\mathfrak{E}_{1}(\lambda)\right) \cdot \mathcal{D}\left(\lambda I_{M}-\mathscr{L}\right) \cdot \mathcal{D}\left(\mathfrak{F}_{1}(\lambda)\right) .
\end{aligned}
$$

Thus, by Theorem 1.1,

$$
\chi\left[\mathfrak{L}, \lambda_{0}\right]=\operatorname{ord}_{\lambda_{0}} \mathcal{D}\left[\mathscr{S}_{\mathfrak{L}\left(\lambda_{0}\right),(P, Q)}(\mathfrak{L}(\lambda))\right]=\operatorname{ord}_{\lambda_{0}} \mathcal{D}\left(\lambda I_{M}-\mathscr{L}\right) .
$$

On the other hand, owing to Theorem [1.3, we already know that

$$
\mathcal{D}\left(\lambda I_{M}-\mathscr{L}\right)=i\left(\mathcal{D}^{-1}(0), \lambda I_{M}-\mathscr{L} ; \lambda_{0} I_{M}-\mathscr{L}\right) .
$$

Therefore,

$$
\chi\left[\mathfrak{L}, \lambda_{0}\right]=i\left(\mathcal{D}^{-1}(0), \lambda I_{M}-\mathscr{L} ; \lambda_{0} I_{M}-\mathscr{L}\right),
$$

which ends the proof of Theorem 1.4 .

\section{REFERENCES}

[1] T. Banachiewicz, Zur Berechnung der Determinanten, wie auch der Inversen, und zur darauf basierten Auflosung der Systeme linearer Gleichungen, Acta Astron. Ser. C 3 (1937), 41-67.

[2] D. Bump, Algebraic Geometry, World Scientific, 1998.

[3] W. L. Chow, On equivalence classes of cycles in an algebraic variety, Ann. of Math. 64 (1956), 450-479.

[4] D. Eisenbud, Commutative Algebra with a View toward Algebraic Geometry, Graduate Texts in Mathematics 150, Springer, 1995.

[5] D. Eisenbud and J. Harris, 3264 and All That: A Second Course in Algebraic Geometry, Cambridge University Press, 2016.

[6] J. Esquinas, Optimal multiplicity in local bifurcation theory, II: General case, J. Diff. Equations 75 (1988), 206-215.

[7] J. Esquinas and J. López-Gómez, Optimal multiplicity in local bifurcation theory, I: Generalized generic eigenvalues, J. Diff. Equations 71 (1988), 72-92.

[8] E. I. Fredholm, Sur une classe d'equations fonctionnelles, Acta Math 27, (1903), 365-390.

[9] W. Fulton, Algebraic Curves: An introduction to Algebraic Geometry, Benjamin-Cummings Publishing Co., Subs. of Addison Wesley Longman, 1969.

[10] I. Göhberg, S. Goldberg and M. A. Kaashoek, Basic Classes of Linear Operators, Springer, Bassel, 2003.

[11] I. C. Göhberg and E. I. Sigal, An Operator Generalization of the Logarithmic Residue Theorem and the Theorem of Rouché, Math. Sbornik 84(126) (1971), 607-629. English Trans.: Math. USSR Sbornik 13 (1971), 603-625.

[12] P. M. Fitzpatrick and J. Pejsachowicz, Parity and generalized multiplicity, Trans. Amer. Math. Soc. 326 (1991), 281-305.

[13] W. Fulton, Intersection Theory, Second Edition, Springer, 1998.

[14] R. Hartshorne, Algebraic Geometry, Graduate texts in mathematics 52, Springer 1977.

[15] J. Ize, Bifurcation Theory for Fredholm Operators, Mem. Amer. Math. Soc. 174, Providence, 1976.

[16] M. A. Krasnoselskij, Topological Methods in the Theory of Nonlinear Integral Equations, Pergamon Press, New York, 1964.

[17] B. Laloux and J. Mawhin, Coincidence index and multiplicity, Trans. Amer. Math. Soc. 217 (1976), 143-162. 
[18] B. Laloux and J. Mawhin, Multiplicity, Leray-Schauder formula and bifurcation, J. Diff. Eqns. 24 (1977), 309-322.

[19] J. López-Gómez, Ecuaciones diferenciales y variable compleja, con teoría espectral y una introducción al grado topológico de Brouwer, Prentice Hall, Madrid, 2001.

[20] J. López-Gómez, Spectral Theory and Nonlinear Functional Analysis, CRC Press, Chapman and Hall RNM 426, Boca Raton, 2001.

[21] J. López-Gómez, Global bifurcation for Fredholm operators, Rend. Istit. Mat. Univ. Trieste 48 (2016), 539-564. DOI: $10.13137 / 2464-8728 / 13172$

[22] J. López-Gómez and C. Mora-Corral, Counting zeroes of $\mathcal{C}^{1}$-Fredholm maps of index zero, Bull. London Math. Soc. 37 (2005) 778-792.

[23] J. López-Gómez and C. Mora-Corral, Algebraic Multiplicity of Eigenvalues of Linear Operators, Operator Theory, Advances and Applications 177, Bassel, 2007.

[24] J. López-Gómez and J. C. Sampedro, Algebraic multiplicity and topological degree for Fredholm operators, Nonlinear Anlaysis (2020) 112019, https://doi.org/10.1016/j.na.2020.112019.

[25] R. J. Magnus, A generalization of multiplicity and the problem of bifurcation, Proc. Lond. Math. Soc. 32 (1976), 251-278.

[26] C. Mora-Corral, On the Uniqueness of the Algebraic Multiplicity, J. London Math. Soc. 69 (2004), 231-242.

[27] L. Nirenberg, Topics in Nonlinear Functional Analysis, Courant Institute of Mathematical Sciences, New York, 1974.

[28] P. J. Rabier, Generalized Jordan chains and two bifurcation theorems of Krasnoselskij, Nonl. Anal. TMA 13 (1989), 903-934.

[29] P. H. Rabinowitz, Some global results for nonlinear eigenvalue problems, J. Funct. Anal. 7 (1971), $487-513$.

[30] J. Serre, Local Algebra, Springer monographs in mathematics, Springer, 2000.

Institute of Interdisciplinary Mathematics (IMI), Department of Analysis and Applied Mathematics, Complutense University of Madrid, 28040-Madrid, Spain.

Email address: Lopez_Gomez@mat.ucm.es, juancsam@ucm.es 